\title{
ON AUTOMORPHISMS OF POLYADIC ALGEBRAS
}

\author{
BY \\ AUBERT DAIGNEAULT( $\left.{ }^{1}\right)$
}

Introduction. This paper belongs to the theory of polyadic algebras as developed by Halmos [11-15], but it has a bearing on the theory of models. Two central concepts are those of homogeneous $\left({ }^{2}\right)$ and of normal extensions of a polyadic algebra (see the beginning of $\$ 2$ and of $\S 6$ ). These concepts bear some resemblance to concepts of the theory of algebraic extensions of fields; however, we have been influenced more immediately by M. Krasner's general Galois theory [33-35] which can be given a polyadic interpretation. Aside from the short preliminary Chapter 0 , the paper is divided into sections grouped into three chapters. Each section begins by an outline of its contents. We proceed to an analysis of the main results of the paper. All polyadic algebras considered are locally finite of infinite degree.

The highlights of Chapter I are: (i) the possibility of extending any simple extension of a (simple polyadic) algebra to a simple homogeneous and normal extension (Corollary 4.6); (ii) a first step on a Galois theory (Theorem 4.5) which, in the case of a full simple functional algebra with finite domain, takes a definite form (Theorem 4.7) closely related to Krasner's theory in that case; (iii) the existence and unicity of $\alpha^{+}$-universal-homogeneous algebras in the sense of B. Jónsson $[21 ; 22]$ in certain classes of polyadic algebras (Theorem 6.3 , Theorem 6.6). The results in (iii) make use of Jónsson's work which is dependent on the continuum hypothesis (see also Theorem 5.5 which is independent of this hypothesis).

Chapter II departs from the theme of automorphisms and uses little aside from Chapter 0 and $\$ 1$. The main result here is Theorem 8.1 which gives a description of the functional representations of a full simple functional algebra in terms of a new generalization of the concept of reduced power.

In Chapter III, we propose to show that the algebraic results of the first two chapters, when put together, embody several known model-theoretic results or new forms of such results. After a section devoted to the connections between Model theory and polyadic algebras, we give new proofs of Beth's theorem

Presented to the Society, January 22, 1962 under the title Extension of isomorphisms of polyadic algebras; received by the editors April 5, 1963.

(1) Some of the results of this paper were announced in [5] and [6]; a few were already contained in the author's doctoral dissertation [4]. Most of the work on this paper has been done while the author was a Fellow of the Summer Research Institute of the Canadian Mathematical Congress in 1961 and 1962.

(2) The use of this word here is unrelated to its meaning in [13]. 
[1] in the theory of definition (Theorem 11.1) and a generalization thereof (Theorem 11.2) due to L. Svenonius [42] $\left({ }^{3}\right)$. It will be seen that this last theorem is related to the concept of normal extension. Next, using our generalized ultrapowers (and without assuming the generalized continuum hypothesis) we obtain a characterization (Theorem 12.1 and Corollary 12.2) of the notion of elementary equivalence similar to those given by S. B. Kochen [30-32] and H. J. Keisler [23-29]. In some respects our characterization is an improvement over the ones of these authors (see discussion following Corollary 12.2). Here it is the concept of homogeneous extension which is put to profit. Finally, we give a proof of a theorem (Theorem 13.1) of Vaught [45].

The concept of locally finite polyadic algebra of infinite degree without equality is used in its full generality: as far as Model theory is concerned this generality enables us to handle simultaneously models of different cardinalities in $\$ 12$. Also the theory of operations in polyadic algebras plays an essential role, especially in Chapter II. The paper presupposes little beyond an acquaintance with Halmos' algebraic logic papers which are now conveniently available in book form [16].

\section{Chapter 0. Preliminaries}

The purpose of this introductory chapter is to set up the notation and terminology to be used throughout and to state some basic known results. All unexplained notation and terminology is to be found in Halmos [11-15].

Unless otherwise noted, the word "algebra" shall always mean a locally finite polyadic algebra of infinite degree. The set of variables $I$ is the same infinite set for all algebras considered; sometimes it will be convenient to assume that $I$ is denumerable. An algebra $(A, I, S, \exists)$ shall be denoted simply by $A$. Our notation for substitutions differs slightly from that of Halmos: we write $S(i / j)$ where Halmos would write $S(j / i)$ for instance.

Small Greek letters will be used to denote ordinal numbers, mappings of several kinds (in particular transformations, i.e., elements of $I^{\boldsymbol{I}}$ ) and types of relational systems. The letter " $\delta$ " is reserved to denote any identity mapping, and the letter " $\omega$ " is reserved to denote the set of all positive integers. Cardinal numbers are identified with initial ordinal numbers. The cardinal next to a cardinal $\alpha$ is denoted by $\alpha^{+}$so that the generalized continuum hypothesis is the statement " $\alpha{ }^{+}=2^{\alpha}$ for all infinite cardinal number $\alpha$." The cardinal number of a set $X$ is denoted by $|X|$.

The restriction of a function $f$ to a subset $X$ of its domain is denoted by $f \mid X$. The set of all $x$ such that $\cdots$ is denoted by $\{x \mid \cdots\}$. The set of all functions from a set $K$ into a set $W$ is denoted by $W^{K}$; the value of $x \in W^{K}$ at $k \in K$ is denoted by $x_{k}$. Except for the case just mentioned the value of a function $f$ at an element $p$ of its domain is denoted by $f(p)$ or by $f p$. Let $\sigma: Y \rightarrow X$ be any map

(3) The author is indebted to W. Craig who kindly brought this theorem to his attention. 
and $H$ be any set; $\sigma$ induces a map $X^{H} \rightarrow Y^{H}$ still denoted by $\sigma$ and defined by the equation $(\sigma x)_{h}=\sigma\left(x_{h}\right)$ for all $x \in X^{H}$ and $h \in H$.

Let $A$ be an algebra and $Y$ a set of constants of $A$. For $p \in A, y \in Y^{I}$ and $J \subset I$ we set by definition

$$
S(y / J) p=S\left(y_{i_{1}} / i_{1}\right) \cdots S\left(y_{i_{n}} / i_{n}\right) p,
$$

where $\left\{i_{1}, \cdots, i_{n}\right\}$ is the intersection of a finite support of $p$ with $J$. It is a simple matter to verify that $S(y / J)$ is a well-defined Boolean endomorphism of $A$. If $B$ is a subalgebra of $A$ we denote by $B(Y)$ the smallest subalgebra of $A$ containing $B$ and closed under all $S(y / J)$ for $y \in Y^{I}$ and $J \subset I$. An extension of $B$ such as $B(Y)$ is called an extension by addition of constants. The endomorphism $S(y / J)$ is also denoted by $y(J)$.

By the center of an algebra $A$ we mean the Boolean algebra $B$ of all closed elements of $A$. The ideals of $B$ correspond biuniquely to the polyadic ideals of $A$. By the quotient of $A$ by an ideal $N$ of $B$ (or by the corresponding filter $\left.N^{\prime}=\left\{p^{\prime} \mid p \in N\right\}\right)$ we mean the same thing as the quotient of $A$ by the polyadic ideal $N=\{p \mid p \in A$ and $\exists(I) p \in N\}$ of $A$. The two-element Boolean algebra $\{0,1\}$ is denoted by 0 . The algebra generated by a set $S$ of predicates of an algebra $A$ is the smallest subalgebra of $A$ containing the range of all predicates in $S$. Tacit use will sometimes be made of a simplified definition of the concept of rich algebra: an algebra $A$ is rich if for every element $p$ of $A$ with a support containing only one variable $i$, there is a constant $c$ of $A$ such that $\exists(i) p=S(c / i) p$. The equivalence of this definition with that of Halmos can be proved by a simple finite induction. Every quotient of a rich algebra is also rich. The value of a constant $c$ at a set $J \subset I$ is sometimes denoted by $c(J)$ instead of $S(c / J)$.

We say that a $B$-valued functional algebra $A$ with domain $X$ is regular if for all $p \in A, x \in X^{I}$ and $J \subset I$ there exists $y \in X^{I}$ such that $x|(I-J)=y|(I-J)$ and $[\exists(J) p](x)=p(y)$, this means that the supremum occurring in the definition of $[\exists(J) p](x)$ is attained.

Let $A$ be a rich algebra with center $B$, and denote by $X$ the set of all constants of $A$. By the canonical representation of $A$ we mean the monomorphism $f$ of $A$ into a $B$-valued functional algebra with domain $X$ and defined as follows: for $p \in A$ and $x \in X^{I}$ we set $(f p)(x)=S(x / I) p$. The image of a rich algebra under its canonical representation is regular.

If $X$ is any set and $B$ is a complete Boolean algebra we denote by $F\left(X^{I}, B\right)$ the full functional algebra of all functions with finite support from $X^{I}$ to $B$. If $B=0$ this algebra is also denoted by $C_{X}$. If $A$ is a simple rich algebra and $X$ is the set of all its constants, we call $C_{X}$ (or an abstract copy of it) the full extension of $A$ when $A$ is imbedded into $C_{X}$ by the canonical representation $f$. Any algebra isomorphic to an $F\left(X^{I}, B\right)$ is said to be full. If $B$ is any Boolean algebra with McNeille completion $\bar{B}$ and if $A$ is a $B$-valued algebra with domain $X$ then $A$ can always be considered as a subalgebra of $F\left(X^{I}, \bar{B}\right)$. 
The following fundamental representation theorem is used tacitly throughout the paper.

THEOREM. Any algebra admits a rich extension and hence a faithful representation by a regular functional algebra, and also a 0-valued representation. If the algebra has an equality $E$, each of these representations maps $E$ on a reduced equality.

For any positive integer, an $n$-ary relation (or predicate) on a set $X$ is any function $X^{n} \rightarrow \mathbf{0}$. A (finitary) type is a function $\mu$ from any set $\Lambda$ to positive integers. A family of relations $\left\{R_{\lambda} \mid \lambda \in \Lambda\right\}$ on a set $X$ is said to be of type $\mu$, if for all $\lambda, R_{\lambda}$ is $\mu(\lambda)$-ary. A (relational) system of type $\mu$ is a couple $\left\langle X,\left\{R_{\lambda} \mid \lambda \in \Lambda\right\}\right\rangle$ consisting of a nonempty set $X$ and of a family of relations of type $\mu$ on $X$. The system will usually be denoted by $\left\langle X, R_{\lambda}\right\rangle$ or more simply by $X$ when no ambiguity will result from this. The cardinality of the system is $|X|$ and the number of relations of the type $\mu$ is $|\Lambda|$. A "distinguished constant" $a$ is assimilated to the characteristic function of the singleton $\{a\}$, and $n$-ary operations are treated as $(n+1)$-ary relations in the usual fashion.

Systems of the same type are said to be similar. If $\left\langle X, R_{\lambda}\right\rangle$ and $\left\langle Y, S_{\lambda}\right\rangle$ are similar, $X$ is a subsystem of $Y$ if $X \subset Y$ and for each $\lambda$ and all $\left(x_{1}, \cdots, x_{\mu(\lambda)}\right) \in X^{\mu(\lambda)}$

$$
R_{\lambda}\left(x_{1}, \cdots, x_{\mu(\lambda)}\right)=S_{\lambda}\left(x_{1}, \cdots, x_{\mu(\lambda)}\right) .
$$

A system $X$ is imbeddable in a system $Y$ if it is isomorphic to a subsystem of $Y$.

As a rule the word "proposition" denotes a statement which is later superseded by another statement. The abbreviation "iff" is used to mean "if and only if." Items such as equations are numbered consecutively, starting afresh in each section.

\section{Chapter I. Automor phisms}

1. Simple full algebras. In this section we study the relations between predicates and operations defined in a set $X$ and predicates and operations of functional algebras with domain $X$. Specializing to the functional algebra $C_{X}$ we establish a correspondence between the automorphisms of that algebra and the permutations of $X$. We conclude with a theorem pertaining to the possibility of extending automorphisms of $C_{X}$ to simple full extensions.

Let $X$ be a (nonempty) set, $n$ a positive integer, and $B$ a Boolean algebra. An $n$-place $B$-valued predicate of $X$ is any function $R: X^{n} \rightarrow B$. If $A$ is a $B$-valued functional algebra with domain $X$ we may define, for each $n$-place predicate $P$ of $A$ a $B$-valued $n$-place predicate

$$
P^{*}\left(x_{1}, \cdots, x_{n}\right)=P\left(i_{1}, \cdots, i_{n}\right)(x)
$$

where $\left(x_{1}, \cdots, x_{n}\right)$ is any element of $X^{n}$, and $\left(i_{1}, \cdots, i_{n}\right) \in I^{n}$ and $x \in X^{I}$ are such that $x_{i_{1}}=x_{1}, \cdots, x_{i}=x_{n}$. Supposing now that $A$ is a full functional algebra 
$F\left(X^{I}, B\right)$ and that $R$ is any $B$-valued $n$-place predicate of $X$, we may in turn define an $n$-place predicate $\hat{R}$ of $A$ by

$$
\hat{R}\left(i_{1}, \cdots, i_{n}\right)(x)=R\left(x_{i_{1}}, \cdots, x_{i_{n}}\right)
$$

for all $\left(i_{1}, \cdots, i_{n}\right) \in I^{n}$ and $x \in X^{I}$. We shall sometimes write $R^{\wedge}$ instead of $R$. With these notations we have for any $B$-valued predicate $R$ of $X, \hat{R}^{*}=R$ and for any predicate $P$ of $A,\left(P^{*}\right)^{\wedge}=P$.

We remind the reader of the connection there is, in any algebra $A$ with equality $E$, between the $n$-place operations $Q$ and the $(n+1)$-place predicates $P$ singlevalued with respect to their last $n$ arguments (i.e., such that for all $\left(i_{1}, \cdots, i_{n}\right) \in I^{n}$ and $\left.i \in I-\left\{i_{1}, \cdots, i_{n}\right\}, \exists !(i) P\left(i, i_{1}, \cdots, i_{n}\right)=1\right)$. The correspondence is one-to-one and is such that

$$
P\left(i, i_{1}, \cdots, i_{n}\right)=E\left[i, Q\left(i_{1}, \cdots, i_{n}\right)\right]
$$

and

$$
S\left[Q\left(i_{1}, \cdots, i_{n}\right) / J\right] p=\exists(k)\left[S(k / J) p \wedge P\left(k, i_{1}, \cdots, i_{n}\right)\right]
$$

for every $\left(i, i_{1}, \cdots, i_{n}\right) \in I^{n+1}, J \subset I$, and $k \notin\left\{i_{1}, \cdots, i_{n}\right\} \cup(L-J)$ where $L$ is a support of $p\left({ }^{4}\right)$.

Turning now to operations in functional algebras, we let again $A$ be a full algebra $F\left(X^{I}, B\right)$. For any $n$-place operation $T$ of $X$ we define an $(n+1)$-place (0-valued) predicate $R$ by

$$
R\left(x, x_{1}, \cdots, x_{n}\right)=1 \text { iff } x=T\left(x_{1}, \cdots, x_{n}\right)
$$

for all $\left(x, x_{1}, \cdots, x_{n}\right) \in X^{n+1}$. It is then easy to show that $\hat{R}$ is single-valued in its last $n$ variables, the implicit reference to equality being to the functional equality $E_{0}$ of $A$. Let $\hat{T}$ be the $n$-place operation of $A$ corresponding to $\hat{R}$. It follows from (4) in which $Q=\hat{T}$ and $P=\hat{R}$ that for all $\left(i_{1}, \cdots, i_{n}\right) \in I_{n}, J \subset I, p \in A$ and $x \in X^{I}$

$$
S\left[\hat{T}\left(i_{1}, \cdots, i_{n}\right) / J\right] p(x)=p(y)
$$

where $y \in X^{I}$ is defined by the conditicns $y_{1}=x_{i}$ if $i \notin J$ and $y_{i}=T\left(x_{i_{1}}, \cdots, x_{i}\right)$ if $i \in J$. The proof of this is a simple exercise which we omit and which uses essentially the 0 -valuedness of $R$.

Similarly, to any $x \in X$ there corresponds a constant $x$ of $F\left(X^{I}, B\right)=A$ determined by the equation

$$
[\hat{x}(J) p](y)=p(z)
$$

where $p \in A, J \subset I, p \in A$ and $y \in X^{I}$, and $z \in X^{I}$ is defined by the conditions $z|(I-J)=y|(I-J)$ and $z_{i}=x$ for all $i \in J$. Indeed $x$ can be treated as the $n$-place operation $T$ of $X$ (for any $n>0$ ) that is identically equal to $x$ on $X^{n}$.

(4) It follows from this that if $A$ is an equality subalgebra of an equality algebra $\bar{A}$, then any operation of $A$ extends uniquely to an operation of $\bar{A}$. We shall not, as a rule, distinguish $A$ between an operation of $A$ and its extension to $\bar{A}$. 
Also any constant $c$ of any algebra $A$ can be treated as the $n$-place operation $Q$ of $A$ which is identically equal to $c$ on $I^{n}$.

It is easy but tedious to verify that if $T, T_{1}, \cdots, T_{n}$ are operations on $X$ and $T$ is $n$-ary then

$$
\left[T\left(T_{1}, \cdots, T_{n}\right)\right]^{\wedge}=\hat{T}\left(\hat{T}_{1}, \cdots, \hat{T}_{n}\right) .
$$

The proof is based on (4) and (5).

If now $A$ is any $B$-valued functional algebra with domain $X$, we say that an $n$-place operation $Q^{*}$ of $X$ represents an $n$-place operation $Q$ of $A$ if for all $\left(i_{1}, \cdots, i_{n}\right) \in I^{n}, J \subset I, p \in A$ and $x \in X^{I}$

$$
S\left[Q\left(i_{1}, \cdots, i_{n}\right) / J\right] p(x)=p(y)
$$

where $y_{i}=x_{i}$ if $i \notin J$ and $y_{i}=Q^{*}\left(x_{i_{1}}, \cdots, x_{i_{n}}\right)$ if $i \in J$. Thus, for instance, in the example of the full algebra $F\left(X^{I}, B\right)$ above, $T$ represents $\hat{T}$. Our next result asserts that if $A$ is suitably restricted, all its operations are uniquely represented by operations of $X$. For this we need a lemma.

Lemma 1.1. If $C$ is an algebra with equality $E$ then $E$ is an equality for any extension of $C$ by addition of constants $C(Y)$.

Proof. The only thing to show is that $E$ is substitutive, i.e., for all $q \in C(Y)$ and $i$ and $j$ in $I, q \wedge E(i, j) \leqq S(i / j) q$. Express $q$ in the form $S(y / J) p$ with $p \in A$, $y \in Y^{I}$ and $J \cap\{i, j\}=\varnothing$. As $S(y / J)$ is an endomorphism, it is monotone. The desired inequality is obtained from the same inequality with $p$ instead of $q$, by applying $S(y / J)$.

Q.E.D.

THEOREM 1.2. Let $A$ be a regular B-valued functional algebra with domain $X$ and with a reduced equality $E$. For any n-place operation $Q$ of $A$ there is a unique n-place operation $Q^{*}$ of $X$ which represents $Q$. If $P$ is the $(n+1)$-place predicate of $A$ corresponding to $Q$ then for all $\left(x, x_{1}, \cdots, x_{n}\right) \in X^{n+1}$,

$$
P^{*}\left(x, x_{1}, \cdots, x_{n}\right)=1 \text { iff } x=Q^{*}\left(x_{1}, \cdots, x_{n}\right) .
$$

If $Q, Q_{1}, \cdots, Q_{n}$ are operations of $A$ such that $Q$ is $n$-ary then

$$
\left[Q\left(Q_{1}, \cdots, Q_{n}\right)\right]^{*}=Q^{*}\left(Q_{1}^{*}, \cdots, Q_{n}^{*}\right) .
$$

Proof. From the fact that $A$ is regular and $E$ is reduced it is easy to see that (10) indeed defines an operation $Q^{*}$. Now letting $Q^{\prime}$ be any operation on $X$ representing $Q$ we have, setting $p=E(i, j)$ in (9) with $Q^{\prime}$ instead of $Q^{*}$ and assuming further that all variables of $\left\{j, i, i_{1}, \cdots, i_{n}\right\}$ are distinct, that $E\left[i, Q\left(i_{1}, \cdots, i_{n}\right)\right](x)=E(i, j)(y)$, which means that

$$
P^{*}\left(x_{i}, x_{i_{1}}, \cdots, x_{i_{n}}\right)=1 \text { iff } x_{i}=Q^{\prime}\left(x_{i_{1}}, \cdots, x_{i_{n}}\right)
$$

for all $x$. Therefore $Q^{\prime}=Q^{*}$ and $Q^{*}$ is the only operation of $X$ that is liable to represent $Q$. 
There remains to prove (9) for the $Q^{*}$ defined by (10). It is sufficient to do that in the case where $J=\{j\}$. Assuming $\left(i_{1}, \cdots, i_{n}\right), p$ and $x$ given we have successively

$$
\begin{aligned}
y_{j} & =Q^{*}\left(x_{i_{1}}, \cdots, x_{i_{n}}\right), P^{*}\left(y_{j}, x_{i_{1}}, \cdots, x_{i_{n}}\right)=1, \\
P\left(\hat{y}_{j}, \hat{x}_{i_{1}}, \cdots, \hat{x}_{i_{n}}\right) & =1, E\left[j, Q\left(\hat{x}_{i_{1}}, \cdots, \hat{x}_{i_{n}}\right)\right]=E\left(j, \hat{y}_{j}\right), \\
\exists(j)\left[p \wedge E\left(j, Q\left(\hat{x}_{i_{1}}, \cdots, \hat{x}_{i_{n}}\right)\right]\right. & =\exists(j)\left[p \wedge E\left(j, \hat{y}_{j}\right)\right], \\
S\left[Q\left(\hat{x}_{i_{1}}, \cdots, \hat{x}_{i_{n}}\right) / j\right] p(x) & =S\left(\hat{y}_{j} / j\right) p(x), \\
S\left[Q\left(i_{1}, \cdots, i_{n}\right) / j\right] p(x) & =p(y) .
\end{aligned}
$$

Equation (11) follows from a direct check that if $Q^{*}, Q_{1}^{*}, \cdots, Q_{n}^{*}$ represent respectively $Q, Q_{1}, \cdots, Q_{n}$, then $Q^{*}\left(Q_{1}{ }^{*}, \cdots, Q_{n}{ }^{*}\right)$ represents $Q\left(Q_{1}, \cdots, Q_{n}\right)$. We omit this verification which is straightforward.

Q.E.D.

If the Boolean algebra $B$ of Theorem 1.2 is complete, then it follows that $\left(Q^{*}\right) \wedge$ is an extension of $Q$ to the full algebra $F\left(X^{I}, B\right)$. As already noted, if $T$ is any operation on $X, T$ represents $\hat{T}$; hence $\hat{T}^{*}=T$. Unless the Boolean algebra $B$ is finite, or else $X$ is finite, the full algebra $F\left(X^{I}, B\right)$ is not regular and Theorem 1.2 does not apply. In general a full algebra has constants which are not represented in the domain. But if $B=\mathbf{0}$, for instance, the correspondence $T \leftrightarrow \hat{T}$ (or $Q^{*} \leftrightarrow Q$ ) between the operations on $X$ and those of $C_{X}$ is one-one. In particular we have the one-one correspondence $x \leftrightarrow \hat{x}$, between $X$ and the set of all constants of $C_{X}$.

If $\sigma: A \rightarrow A$ is an homomorphism of polyadic algebras and $P$ is an $n$-place predicate of $A$, the image of $P$ under $\sigma$ is an $n$-place predicate $\sigma P$ of $\bar{A}$ defined by the equation

$$
(\sigma P)\left(i_{1}, \cdots, i_{n}\right)=\sigma\left[P\left(i_{1}, \cdots, i_{n}\right)\right] .
$$

Unless $\sigma$ is surjective ((15.3) in [13]) it is not possible to define the image of an operation in general. We next prove a lemma that asserts that in the case of equality algebras everything works fine. The lemma is easy and its proof is again based on the correspondence between operations and single-valued predicates.

Lemma 1.3. Let $A$ and $A$ be equality algebras and $\sigma: A \rightarrow A$ be an equality homomorphism. For any $n$-ary operation $T$ of $A$ there is a unique operation $\sigma T$ of $\bar{A}$ such that for all $\left(i_{1}, \cdots, i_{n}\right) \in I^{n}, J \subset I$, and $p \in A$

$$
\sigma\left[S\left(T\left(i_{1}, \cdots, i_{n}\right) / J\right) p\right]=S\left[(\sigma T)\left(i_{1}, \cdots, i_{n}\right) / J\right] \sigma p .
$$

Moreover, if $T, T_{1}, \cdots, T_{n}$ are operations of $A$ with $T$ of degree $n$

$$
\sigma\left[T\left(T_{1}, \cdots, T_{n}\right)\right]=(\sigma T)\left(\sigma T_{1}, \cdots, \sigma T_{n}\right) .
$$


Proof. Let $E$ and $\bar{E}$ be the equalities of $A$ and $\bar{A}$ respectively. Let $R$ be the $(n+1)$-place predicate of $A$ corresponding to $T$. Since $R$ is single-valued (with respect to its last $n$ arguments) and $\sigma E=\bar{E}, \sigma R$ is also single-valued. Let $\sigma T$ be the operation of $A$ corresponding to $\sigma R$. Equation (13) follows immediately from (4) applied to the couples $(T, R)$ and $(\sigma T, \sigma R)$ instead of $(Q, P)$. To show unicity we let $T^{\prime}$ be any $n$-place operation of $A$ such that (13) is satisfied with $T^{\prime}$ instead of $\sigma T$. Letting $p=E\left[i, T\left(i_{1}, \cdots, i_{n}\right)\right]$ and $J=\{i\}$ with $i \notin\left\{i_{1}, \cdots, i_{n}\right\}$ in this equation and in (13) we get $1=E\left[T\left(i_{1}, \cdots, i_{n}\right), T\left(i_{1}, \cdots, i_{n}\right)\right]$ $=\bar{E}\left[T^{\prime}\left(i_{1}, \cdots, i_{n}\right), \sigma T\left(i_{1}, \cdots, i_{n}\right)\right]$, and hence $T^{\prime}=\sigma T$.

As to equation (14), consider, say, the case where $T$ and $T_{2}$ are binary and $T_{1}$ unary and set $T_{0}=T\left(T_{1}, T_{2}\right)$. Then (14) follows easily from the way $\sigma T$ has been defined above and the equation

$$
E\left[i, T_{0}\left(i_{1}, i_{2}, i_{3}\right)\right]=\exists\left(j_{1}\right) \exists\left(j_{2}\right)\left[E\left(j_{1}, T_{1}\left(i_{1}\right)\right) \wedge E\left(j_{2}, T_{2}\left(i_{2}, i_{3}\right)\right) \wedge E\left(i, T\left(j_{1}, j_{2}\right)\right)\right]
$$

together with its analogue with the $\sigma T$ 's instead of the T's and $\bar{E}$ instead of $E$.

Q.E.D.

We shall sometimes distinguish between $\sigma$ and the mapping of operations $\bar{\sigma}$ that it induces; thus we shall write $\bar{\sigma} T$ instead of $\sigma T$. If, for instance, $\sigma$ is an isomorphism, we have, for every constant $c$ of $A$, element $q$ of $A$ and set $J \subset I$, $(\bar{\sigma} c)(J) q=\sigma c(J) \sigma^{-1} q$. If $Y$ is a set of constants of $A, c \in Y^{I}$ and again $J \subset I$, then we still have $(\bar{\sigma} c)(J)=\sigma c(J) \sigma^{-1}$. The verification of this is immediate.

When $\sigma$ is an automorphism of an algebra $A$ we may think of $\bar{\sigma}$ as a permutation of the set $K$ of all constants $c$ of $A$. Then it follows easily from the equation $(\bar{\sigma} c)(J)=\sigma c(J) \sigma^{-1}$ that the mapping $\sigma \rightarrow \bar{\sigma}$ is an homomorphism of the group of all automorphisms of $A$ into the group of all permutations of $K$. Thus, in particular, $\overline{\sigma^{-1}}=(\bar{\sigma})^{-1}$.

Now we consider the special case of the automorphisms $\sigma$ of $C_{X}$. First, some notation. For any subset $Y$ of $X$ we let $\hat{Y}=\{\hat{y} \mid y \in Y\}$. If $y \in Y^{I}$ then $\hat{y} \in \hat{Y^{I}}$ has the obvious meaning. With this notation we have, for instance, $\hat{x}(I) p=p(x)$ for all $p \in C_{X}$ and $x \in X^{I}$. We denote by $\tilde{\sigma}$ the permutation of $X$ corresponding to the permutation $\bar{\sigma}$ of $\hat{X}$; this means that, for $x \in X, \tilde{\sigma} x$ is the element $(\bar{\sigma} \hat{x})^{*}$ of $X$ corresponding to the constant $\bar{\sigma} \hat{x}$ of $C_{X}$.

THEOREM 1.4. The mapping $\sigma \rightarrow \tilde{\sigma}$ is an isomorphism of the group of all automorphisms of $C_{X}$ onto the group of all permutations of $X$.

Proof. For all $\hat{x} \in \hat{X}^{I}$ and $q \in C_{X}$ we have

$$
(\bar{\sigma} \hat{x})(I) q=\sigma \hat{x}(I) \sigma^{-1} q .
$$

Since $\hat{x}(I) \sigma^{-1} q$ is a closed element of $C_{X}$, it is 0 or 1 and therefore invariant under $\sigma$. Hence (15) is equivalent to

$$
q(\tilde{\sigma} x)=\left(\sigma^{-1} q\right)(x)
$$


which, in turn, is seen to be equivalent to

$$
(\sigma p)(x)=p\left(\tilde{\sigma}^{-1} x\right)
$$

by setting $q=\sigma p$. From (17) it follows that $\sigma$ is determined by $\tilde{\sigma}$. Therefore the homomorphism $\sigma \rightarrow \tilde{\sigma}$ is an isomorphism.

Also it is easy to check that if $\tilde{\sigma}$ is any permutation of $X$ and $\sigma$ is defined by (17), then $\sigma$ is an automorphism of $C_{X}$.

Q.E.D.

We conclude with a theorem which will be used repeatedly in the sequel and which presents an interest of its own.

THeOREM 1.5. An automorphism $\sigma$ of a simple full algebra $A$ extends uniquely to any simple full extension $B$ of $A$ with the same equality.

Proof. The theorem is equivalent to the following statement. Let $A=C_{X}$, $B=C_{Y}, \rho: A \rightarrow B$ be an equality monomorphism and $\sigma$ be an automorphism of $A$. Then there exists an automorphism $\sigma_{1}$ of $B$ such that the following diagram

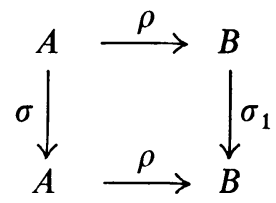

is commutative. Denote by $E$ and $\bar{E}$ the equalities of $A$ and $B$ respectively.

Let $\tilde{\sigma}$ be the permutation of $X$ determined by $\sigma ; \tilde{\sigma}$ induces a (unary) operation $\hat{\sigma}$ of $A$. Set $\hat{\sigma}_{1}=\rho \hat{\sigma}$; this is an operation of $B$ to which corresponds a permutation $\tilde{\sigma}_{1}=(\rho \hat{\sigma})^{*}$ of $Y$. Finally, $\tilde{\sigma}_{1}$ induces the required automorphism $\sigma_{1}$ of $B$.

Now the details. If $q \in A$ and $\left\{i_{1}, \cdots, i_{n}\right\}$ supports $q$ we set by definition

$$
S(\hat{\sigma}) q=S\left[\hat{\sigma}\left(i_{1}\right) / i_{1}\right] \cdots S\left[\hat{\sigma}\left(i_{n}\right) / i_{n}\right] q ;
$$

from (6) it follows that for all $q \in A$ and $x \in X^{I}$,

$$
[S(\hat{\sigma}) q](x)=q(\tilde{\sigma} x)
$$

and hence from (16),

$$
S(\hat{\sigma}) q=\sigma^{-1} q .
$$

We have, since $\tilde{\sigma}$ is a permutation $(\forall j) \exists !(i) E[j, \hat{\sigma}(i)]=1$ (for $j \neq i$ ) and hence, since $\rho$ is equality preserving, $(\forall j) \exists !(i) E\left[j, \hat{\sigma}_{1}(i)\right]=1$. Therefore $\tilde{\sigma}_{1}$ is a permutation of $Y$.

To check that $\sigma_{1} \rho=\rho \sigma$ we let $q \in A$ and $y \in Y^{I}$. We have indeed

$$
\begin{aligned}
\left(\sigma_{1} \rho q\right)(y) & =(\rho q)\left(\tilde{\sigma}_{1}^{-1} y\right) & & {[\text { by }(17)] } \\
& =\left[S\left(\hat{\sigma}_{1}^{-1}\right) \rho q\right](y) & & {[\text { by }(19)] } \\
& =\left[\rho S\left(\hat{\sigma}^{-1}\right) q\right](y) & & {\left[\text { since } \hat{\sigma}_{1}=\rho \hat{\sigma}\right] } \\
& =(\rho \sigma q)(y) & & {[\text { by }(20)] . }
\end{aligned}
$$


To prove the unicity part of the theorem we let $\sigma_{1}$ be any automorphism of $B$ such that $\sigma_{1} \rho=\rho \sigma$. From the last sequence of equations we see, working from both ends, that $S\left(\hat{\sigma}_{1}^{-1}\right) \rho=\rho S\left(\hat{\sigma}^{-1}\right)$ which means that $\rho \hat{\sigma}^{-1}=\hat{\sigma}_{1}^{-1}$ or $\hat{\sigma}_{1}=\rho \hat{\sigma}$.

Q.E.D.

No such theorem is available for nonsimple full algebras and this is the reason for which some theorems below are restricted to simple algebras.

2. Homogeneous extensions. By an homogeneous extension of an algebra $A$ we mean an extension $B$ such that any monomorphism of any subalgebra of $A$ into $B$ extends (in at least one way) to an automorphism of $B$. The (second) main result (Theorem 2.11) of this section is the statement that any simple algebra admits a simple rich homogeneous extension. The question of the "unicity" of this extension as well as considerations special to equality algebras and also cardinality considerations are kept for later sections. The first main result (Theorem 2.7) of the present section can be formulated (somewhat vaguely) thus: any two extensions of an algebra can be imbedded into a single extension. This is a crucial step in the application of Jónsson's work to polyadic algebras.

e begin by describing some special concepts and notations. By a pair we shall mean an ordered couple $(D, X)$ where $D$ is an algebra and each element of $X$ acts as a constant of $D$. For instance, $X$ could be a set of constants of an extension of $D$ under which $D$ is closed (and we could in fact assume that, in the definition). The point of novelty here is that it is possible for two distinct elements of $X$ to induce the same constant of $D$. The value of the constant corresponding to $x \in X$ at a set $J \subset I$ will be denoted by $S(x / J)$, or $x(J)$, as if $x$ itself were the constant and for $x \in X^{I}, S(x / J)$ is defined as in the case of constants. If $A$ is a subalgebra of $D$ and $Y \subset X, A(Y)$ will denote the subalgebra of $D$ generated by $A$ and the constants of $D$ induced by the elements of $Y$. The pair $(A(Y), Y)$ is also denoted by $A[Y]$; thus the algebra of a pair such as $A[Y]$ is not $A$ but $A(Y)$. By a subpair of a pair $(D, X)$ we mean a pair $\left(D_{1}, X_{1}\right)$ such that $D_{1}$ is a subalgebra of $D$ and $X_{1} \subset X$. Equivalently we say that $(D, X)$ is an extension of $\left(D_{1}, X_{1}\right)$.

An homomorphism of a pair $\left(D_{1}, X_{1}\right)$ into a pair $\left(D_{2}, X_{2}\right)$ is a couple $(\sigma, \bar{\sigma})$ where $\sigma$ is an homomorphism of $D_{1}$ into $D_{2}$ and $\bar{\sigma}$ is a mapping from $X_{1}$ into $X_{2}$ such that

$$
\sigma[S(x / J) p]=S(\bar{\sigma} x / J) \sigma p
$$

for all $p \in D_{1}, x \in X_{1}^{I}$ and $J \subset I$. The homomorphism $(\sigma, \bar{\sigma})$ is a monomorphism if both $\sigma$ and $\bar{\sigma}$ are injective. The homomorphism $(\sigma, \bar{\sigma})$ is an isomorphism if both $\sigma$ and $\bar{\sigma}$ are bijective.

If $(D, X)$ is a pair and $N$ is an ideal of $D$ then by the natural homomorphism with kernel $N$ we mean the homomorphism $(\sigma, \delta)$ of $(D, X)$ into the pair $(D / N, X)$ defined as follows. First $\sigma$ is the natural homomorphism of $D$ onto $D / N ; \delta$ is 
the identity map of $X$ onto itself; and any $x \in X$ acts on $D / N$ as the constant arising from the constant of $D$ that $x$ induces (see Lemma 15.3 of [13]). Then (1) is satisfied.

The union of an increasing family of pairs has an obvious meaning: if, for instance $\left\{C\left[Y_{i}\right] \mid i=1,2, \cdots\right\}$ is increasing and $Y=\bigcup_{i=1}^{\infty} Y_{i}$, the union pair may be denoted by $C[Y]$.

A pair $(D, X)$ is rich if each element of $D$ has a witness made up of constants induced by elements of $X$. Any pair $\left(D_{1}, X_{1}\right)$ extends to a rich pair $(D, X)$. This follows essentially from the fact that any algebra $D_{1}$ can be extended to a rich algebra $D$ such that every constant of $D_{1}$ can be extended to a constant of $D$ (see Lemma 15.3 in [13]). If $\left(D_{1}, X_{1}\right)$ is simple, $(D, X)$ may be assumed simple.

If $(D, X)$ is a rich pair, we have a corresponding representation $f: D \rightarrow C_{X}$ defined by the equation $(f p)(x)=S(x / I) p$ where $p \in D$ and $x \in X^{I}$. Thus if $D$ is a rich algebra the set of all constants of which is $X$, the representation of $D$ corresponding to $(D, X)$ is the same as the canonical representation of $D$.

In what follows it will be convenient to give preference to the "bracket" notation over the "ordered couple"' notation to denote pairs. Any pair $(D, X)$ can also be denoted by $D[X]$.

LemMA 2.1. Let $A[Z]$ be a subpair of a pair $C[X]$ and let $(\sigma, \bar{\sigma}): A[Z] \rightarrow C[X]$ be a monomorphism. Then $C[X]$ admits an extension $C[Y]$ such that $(\sigma, \bar{\sigma})$ extends to a monomorphism $(\rho, \bar{\rho}): A[X] \rightarrow C[Y]$. Of course, if $C(X)$ is simple, $C(Y)$ may also be assumed to be simple and, in any case, $C[Y]$ can be assumed to be rich.

Proof. Let $t:(X-Z) \rightarrow K$ be a biunique mapping, $K$ being some new set. From the $(I \cup K)$-dilation of $C(X)$, fixing the variables of $K$ we form the pair $C[X \cup K]$ extension of $C[X]$. Every element of $A(X)$ has the form $S(x / J) p$ with $p \in A(Z), J \subset I$ and $x \in(X-Z)^{I}$. Let $N$ be the ideal of $C(X \cup K)$ generated by

$$
\left\{S(t x / I) \sigma p \mid x \in(X-Z)^{I}, p \in A(Z) \text { and } S(x / I) p=0\right\} .
$$

Assuming for the moment that $N$ is proper, we let $(\beta, \bar{\beta}): C[X \cup K] \rightarrow C[Y]$ be an homomorphism with kernel $N$ and such that $\bar{\beta}$ is biunique. Now we define $(\rho, \bar{\rho}): A[X] \rightarrow C[Y]$ by the equations $\bar{\rho}|Z=\bar{\beta} \bar{\sigma}, \bar{\rho}|(X-Z)=\bar{\beta} t$ and

$$
\rho[S(x / J) p]=S(\bar{\rho} x / J) \beta \sigma p
$$

where $p \in A(Z)$ and $x \in(X-Z)^{I}$. It is easy but tedious to check that $\rho$ is well defined and that $(\rho, \bar{\rho})$ is a monomorphism. We, of course, have $\rho \mid A(Z)=\beta \sigma$. We obtain the following commutative diagram in which the unnamed mappings are identity mappings: 


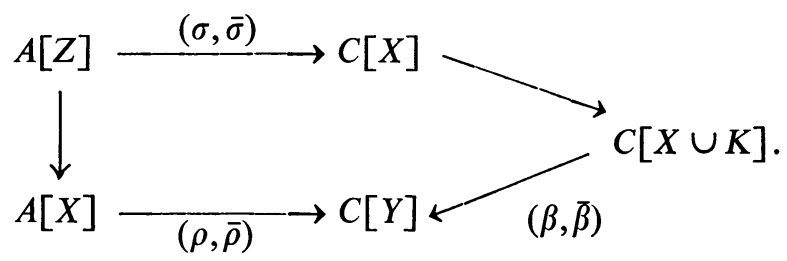

Since $\bar{\beta}$ was supposed biunique, the diagram will express the desired conclusion provided we can show that $N \cap C(X)=\{0\}$. We proceed to do that, noting that this will show at the same time that $N$ is proper. The set of generating elements of $N$ is easily seen to be closed under union and so an element $q$ of $C(X \cup K)$ is in $N$ iff $q$ is $\leqq$ an element of the generating set. Let $q$ be a closed element of $C(X)$ and suppose that $q \leqq S(t x / I) \sigma p$ with $p \in A(Z), x \in(X-Z)^{I}$ and $S(x / I) p=0$. Then

$$
q \leqq \forall(I) \sigma q=\sigma[\forall(I) p] \leqq \sigma[S(x / I) p]=0 .
$$

Q.E.D.

The proof of the next lemma makes use of an idea which has been used repeatedly in the literature; see for instance $[32 ; 38 ; 42]$.

LeMma 2.2. Let $A_{1}\left[X_{1}\right]$ and $A_{2}\left[X_{2}\right]$ be subpairs of a pair $C[X]$ and $(\sigma, \bar{\sigma}): A_{1}\left[X_{1}\right] \rightarrow A_{2}\left[X_{2}\right]$ be an isomorphism. Then there exists a rich extension pair $C[Y]$ of $C[X]$ and an isomorphism $(\rho, \bar{\rho}): A_{1}[Y] \rightarrow A_{2}[Y]$ which extends $(\sigma, \bar{\sigma})$. Moreover, if $C(X)$ is simple, $C(Y)$ may be chosen simple.

Proof. We apply the preceding lemma $\omega$ times to obtain an infinite sequence of monomorphisms of pairs:

$$
\begin{aligned}
(\sigma, \bar{\sigma}): A_{1}\left[X_{1}\right] \rightarrow C\left[X_{2}\right] ; & \left(\sigma_{1}^{-1}, \bar{\sigma}_{1}^{-1}\right): A_{2}\left[Y_{0}\right] \rightarrow C\left[Y_{1}\right] ; \\
\left(\sigma_{2}, \bar{\sigma}_{2}\right): A_{1}\left[Y_{1}\right] \rightarrow C\left[Y_{2}\right] ; & \left(\sigma_{3}^{-1}, \bar{\sigma}_{3}^{-1}\right): A_{2}\left[Y_{2}\right] \rightarrow C\left[Y_{3}\right] ; \\
\left(\sigma_{4}, \bar{\sigma}_{4}\right): A_{1}\left[Y_{3}\right] \rightarrow C\left[Y_{4}\right] ; & \left(\sigma_{5}^{-1}, \bar{\sigma}_{5}^{-1}\right): A_{2}\left[Y_{4}\right] \rightarrow C\left[Y_{5}\right]
\end{aligned}
$$

and so on. The sequence is obtained thus. First, $C\left[Y_{0}\right]$ is any rich extension of $C[X]$. Then we apply Lemma 2.1 to the monomorphism $\left(\sigma^{-1}, \bar{\sigma}^{-1}\right): A_{2}\left[X_{2}\right] \rightarrow C\left[Y_{0}\right]$ to get $\left(\sigma_{1}^{-1}, \bar{\sigma}_{1}^{-1}\right)$. Then we apply Lemma 2.1 to $\left(\sigma_{1}, \bar{\sigma}_{1}\right): A_{1}\left[\bar{\sigma}_{1}^{-1} Y_{0}\right] \rightarrow C\left[Y_{1}\right]$ to get $\left(\sigma_{2}, \bar{\sigma}_{2}\right)$. And so on.

The $C\left[Y_{i}\right]$ form an increasing chain of pairs and we let $C[Y]$ be the union pair. The mapping $(\rho, \bar{\rho})$ will also be the union of the $\left(\sigma_{i}, \bar{\sigma}_{i}\right)$. More precisely, if $q \in A_{1}(Y)$ is in $A_{1}\left(Y_{i}\right)$ and $y \in Y$ is in $Y_{i}$ we set by definition, $\rho(q)=\sigma_{i+1}(q)$ and $\bar{\rho}(y)=\bar{\sigma}_{i+1}(y)$. Since, for all $i,\left(\sigma_{i+1}, \bar{\sigma}_{i+1}\right)$ extends $\left(\sigma_{i}, \bar{\sigma}_{i}\right),(\rho, \bar{\rho})$ is well defined; and since each $\left(\sigma_{i}, \bar{\sigma}_{i}\right)$ is a monomorphism, so is $(\rho, \bar{\rho})$. The mapping $\rho$ is surjective, for an element of $A_{2}[Y]$ is in $A_{2}\left[Y_{i}\right]$ for some $i$, and is therefore in the range of $\sigma_{i+1}$ since it is in the domain of definition of $\sigma_{i+1}^{-1}$. Similarly, $\bar{\sigma}$ is surjective. If $C(X)$ is simple, we obtain a simple $C(Y)$ by selecting a simple $C\left(Y_{i}\right)$ for each $i$.

Q.E.D. 
COROLlary 2.3. For any algebra $C$ and automorphism $\sigma$ of $C$ there exists a rich extension $C_{1}$ of $C$ to which $\sigma$ extends. $C_{1}$ can be obtained from $C$ by addition of constants. If $C$ is simple, $C_{1}$ may also be selected simple.

Proof. This follows immediately from Lemma 2.2 in which we let $A_{1}=A_{2}=C$ and $X_{1}=X_{2}=X=\varnothing$.

Q.E.D.

The main theorem of this section begins to take shape in our next result.

Proposition 2.4. Let $\sigma: A_{1} \rightarrow A_{2}$ be an isomorphism between subalgebras of a simple algebra $C$. Then there exists a simple rich extension $D$ of $C$ such that $\sigma$ extends to an automorphism of $D$.

Proof. We apply Lemma 2.2 (in which $X_{1}=X_{2}=X=\varnothing$ ) and let $D=C_{Y}$. Let also $\rho^{\prime}$ be the automorphism of $C_{Y}$ induced by $\bar{\rho}$ and let $f: C(Y) \rightarrow C_{Y}$ be the representation of $C(Y)$ corresponding to the pair $C[Y]$. The proposition will be proved if the diagram

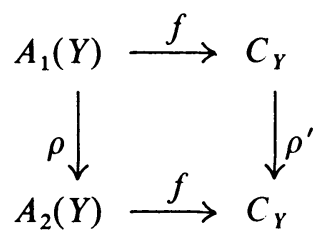

is commutative. To verify this we let $q \in A_{1}(Y)$ and $y \in Y^{I}$. We have

$$
\begin{aligned}
\left(\rho^{\prime} f q\right)(y) & =(f q)\left(\bar{\rho}^{-1} y\right) \quad[\text { by }(17), \S 1] \\
& =S\left(\bar{\rho}^{-1} y / I\right) q .
\end{aligned}
$$

This last expression is a closed element of the simple algebra $A_{1}(Y)$ and is thus left fixed by $\rho$. Applying $\rho$ to it we get [by (1)], $S(y / I) \rho q=(f \rho q)(y)$. Q.E.D.

In the above proof, if we let $A_{1}=A_{2}=C(Y)$ we obtain the following

COROLlary 2.5. An automorphism of a rich simple algebra extends uniquely to the canonical full extension of that algebra.

To prove unicity we let $\rho^{\prime}$ be any automorphism that makes the above diagram commutative and we let $\tilde{\rho}$ be the corresponding permutation of $Y$. It follows using the equations at the end of the proof of Proposition 2.4 that $\tilde{\rho}=\bar{\rho}$.

We shall not consider pairs any more (except in Theorem 4.2) as these have already served their main purpose (in the proof of Proposition 2.4). Some of our results, for instance Proposition 2.4 and Corollary 2.5 above and Theorem 2.7 below, could have been stated for pairs instead of algebras but as we will have almost no use for such generality we shall restrict ourselves to the more familiar concepts. Our proofs can be generalized easily using, this time, Lemma 2.2 in all its generality. 
Proposition 2.4 will be generalized to nonsimple algebras later in this section. Corollary 2.5 could also be generalized but this will not be done in the present paper.

Let $A=F\left(X^{I}, B\right)$ and $A_{1}=F\left(X_{1}^{I}, B\right)$ be full functional algebras with the same center, and let $t: X \rightarrow X_{1}$ be a surjective map. A mapping $s: A_{1} \rightarrow A$ can be defined by setting $(s p)(x)=p(t x)$ for all $p \in A_{1}$ and $x \in X^{I}$. The mapping $s$ is indeed a polyadic monomorphism; the verification of this is straightforward and is a special case of the proof of Lemma 6.4 in [15]. We call a monomorphism such as $s$ a stretch imbedding. It is easy to verify that $s$ is an equality imbedding iff $t$ is biunique. This concept enables us to prove a fundamental property of polyadic algebras which we now need and which will also be used in Chapter III.

THEOREM 2.6. Any two polyadic algebras, indeed any family of such algebras, can be imbedded into a single polyadic algebra.

Proof. Let $\left\{A_{h} \mid h \in H\right\}$ be the given family of polyadic algebras. Let, for each $h, g_{h}: A_{h} \rightarrow F\left(X_{h}^{I}, B_{h}\right)$ be an imbedding into a full functional algebra. All we need to have now is a single $X$ and a single $B$ instead of one for each $h$. Let $B$ be, say, the free product of the $B_{h}$ as $|I|$-complete Boolean algebras [39]. Since all relevant suprema are preserved in the imbeddings $B_{h} \rightarrow B$ we obtain polyadic imbeddings $f_{h}: F\left(X_{h}^{I}, B_{h}\right) \rightarrow F\left(X_{h}^{I}, B\right)$. Now let $X$ be a set such that $|X| \geqq\left|X_{h}\right|$ for all $h$, and for each $h$, let $t_{h}: X \rightarrow X_{h}$ be a surjective map. These maps yield stretch imbeddings $m_{h}: F\left(X_{h}^{I}, B\right) \rightarrow F\left(X^{I}, B\right)$. Finally we obtain the imbeddings $m_{h} f_{h} g_{h}: A_{h} \rightarrow F\left(X^{I}, B\right)$.

Q.E.D.

We are now ready for the first main result of this section.

TheOREM 2.7. Let $f_{i}: A \rightarrow B_{i}, i=1,2$, be polyadic monomorphisms; then there exist polyadic monomorphisms $g_{i}: B_{i} \rightarrow D$ which amalgamate the $f_{i}$, i.e., such that the diagram

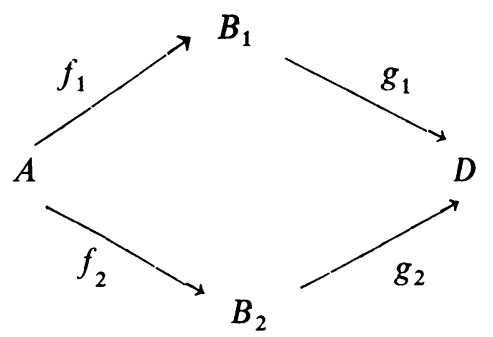

is commutative. Of course, if the $B_{i}$ are simple, $D$ can be selected simple.

Proof. We first deal with the case of simple algebras. Let, by Theorem 2.6, $\sigma_{i}: B_{i} \rightarrow C, i=1,2$, be monomorphisms into a simple algebra $C$. Set $A_{i}=\sigma_{i} f_{i}(A)$ and $\sigma=\left(\sigma_{2} f_{2}\right)\left(\sigma_{1} f_{1}\right)^{-1} ; \sigma$ is an isomorphism of $A_{1}$ onto $A_{2}$. Let $D$ be a simple extension of $C$ such that $\sigma$ extends to an automorphism $\rho$ of $D$ (Proposition 2.4). 
Let $g_{2}=\rho^{-1} \sigma_{2}$ and $g_{1}=\sigma_{1}$. Then for $p \in A$ we have indeed $g_{2} f_{2}(p)=\sigma^{-1} \sigma_{2} f_{2}(p)$ $=\sigma_{1} f_{1}(p)$. This concludes the proof in the simple case.

The general case follows easily from the simple case and from the semisimplicity of polyadic algebras. For convenience we assume that the $f_{i}$ are identity maps. Let $\left\{\left(M_{1 k}, M_{2 k}\right) \mid k \in K\right\}$ be the set of all couples such that for all $k \in K, M_{1 k}$ and $M_{2 k}$ are polyadic maximal ideals of $B_{1}$ and $B_{2}$ respectively such that $M_{1 k} \cap A=M_{2 k} \cap A$. For each $k$, let $g_{i k}: B_{i} \rightarrow D_{k}$ be homomorphisms with kernels, $M_{i k}, i=1,2$. Let $D$ be the algebra of all elements with finite support of the (possibly not locally finite) direct product algebra $\prod D_{k}$ and set for $p \in B_{i}, g_{i}(p)=\left\{g_{i k}(p)\right\}$. Clearly the $g_{i}$ are homomorphisms such that $g_{1}\left|A=g_{2}\right| A$. To show that $g_{1}$, say, is injective we let for $p \in B_{1}, M_{1 k}$ be a maximal ideal of $B_{1}$ such that $p \notin M_{1 k}$ and $M_{2 k}$ be a maximal ideal of $B_{2}$ containing $M_{1 k} \cap A$. Then $g_{1 k}(p) \neq 0$ and hence $g_{1}(p) \neq 0$.

Q.E.D.

When the $B_{i}$ are equal to a single algebra $C$ we may state the result as follows.

Corollary 2.8. Let $A$ be a subalgebra of an algebra $C$ and let $\sigma: A \rightarrow C$ be a monomorphism. Then $C$ admits an extension $D$ such that $\sigma$ extends to a monomorphism $\rho: C \rightarrow D$.

Proof. This follows immediately from Theorem 2.7 in which $B_{1}=B_{2}=C, f_{1}$ is the identity map, $f_{2}=\sigma$ and $C$ is identified to a subalgebra of $D$ by $g_{2}$. Q.E.D.

The unscrupulous reader who pays no particular attention to the continuum hypothesis can leave out the rest of this section as these results will be superseded in $\$ 6$ at the cost of using the continuum hypothesis.

From Corollary 2.3 and Corollary 2.8 it is possible to prove Proposition 2.4 without the hypothesis of simplicity.

THEOREM 2.9. Proposition 2.4 holds in general for nonsimple algebras.

Proof. We use the same device as in the proof of Lemma 2.2. We apply Corollary $2.8 \omega$ times to obtain an infinite sequence of monomorphisms:

$$
\begin{array}{ll}
\sigma: A_{1} \rightarrow C, & \sigma_{1}^{-1}: C \rightarrow C_{1}, \\
\sigma_{2}: C_{1} \rightarrow C_{2}, & \sigma_{3}^{-1}: C_{2} \rightarrow C_{3}, \\
\sigma_{4}: C_{3} \rightarrow C_{4}, & \sigma_{5}^{-1}: C_{4} \rightarrow C_{5}, \text { and so on. }
\end{array}
$$

Each monomorphism in this sequence is obtained by applying Corollary 2.8 to the inverse of the monomorphism that precedes it: thus, $\sigma_{1}^{-1}$ is obtained from $\sigma^{-1}: A_{2} \rightarrow C, \sigma_{2}$ from $\sigma_{1}: \sigma_{1}^{-1}(C) \rightarrow C_{1}$ and so on. Hence, for all $i, \sigma_{i+1}$ extends $\sigma_{i}$. We let $C^{\prime}=\bigcup_{i=1}^{\infty} C_{i}$ and $\rho$ be the common extension of all $\sigma_{i}$; it is obvious that $\rho$ is an automorphism of $C^{\prime}$. Finally, applying Corollary 2.3 with $C^{\prime}$ in the role of $C$, we let $D$ be a rich extension of $C^{\prime}$ to which $\rho$ extends. Q.E.D.

To conclude we generalize Proposition 2.4 in a different direction to get the second main result of this section. 
We shall use tacitly, here and in some later proofs, the following lemma which is easily established, say by Zorn's lemma.

LeMma 2.10. Let $\left\{D_{n} \mid h \in H\right\}$ be an increasing chain of algebras, $H$ being a well-ordered set, such that any automorphism of any $D_{h}$ extends (in at least one way) to an automorphism of the next $D_{h}$. Then any such automorphism extends to the union of the $D_{h}$.

THEOREM 2.11. Any simple algebra $C$ admits a rich simple homogeneous extension $D$.

Proof. We first prove the weaker statement: there exists a rich simple extension $D^{\prime}$ of $C$ such that any monomorphism of any subalgebra of $C$ into $C$ extends to an automorphism of $D^{\prime}$. We obtain $D^{\prime}$ by a transfinite process. Let $\Sigma$ be the set of all monomorphisms $\sigma$ of any subalgebra of $C$ into $C$, arbitrarily well-ordered. We construct a chain $\left\{D_{\sigma} \mid \sigma \in \Sigma\right\}$ of simple full extensions of $C$ such that whenever $\sigma^{\prime}<\sigma$ then $D_{\sigma^{\prime}} \subset D_{\sigma}$ and $\sigma^{\prime}$ extends to an automorphism of $D_{\sigma}$ (in at least one way). If $D_{\sigma^{\prime}}$ has been constructed and $\sigma$ is the successor of $\sigma^{\prime}$ in the ordering of $\Sigma$, we obtain $D_{\sigma}$ by applying Proposition 2.4 with $D_{\sigma^{\prime}}$ in the role of $C$ and $\sigma^{\prime}$ in the role of $\sigma$, to obtain an algebra $D^{*}$, say, and then by letting $D_{\sigma}$ be the canonical full extension of $D^{*}$. The monomorphism $\sigma^{\prime}$ extends to an automorphism of $D_{\sigma}$ by Corollary 2.5 since it extends to an automorphism of $D^{*}$. Also any monomorphism preceding $\sigma^{\prime}$ extends to an automorphism of $D_{\sigma}$ (by Theorem 1.5) since it extends to an automorphism of $D_{\sigma^{\prime}}$.

If $\sigma$ has no predecessor we obtain $D_{\sigma}$ from the $D_{\sigma^{\prime}}$ for $\sigma^{\prime}<\sigma$ by taking the union of these and then taking the canonical full extension of this union. At the end $D^{\prime}$ is obtained simply by taking the union of all $D_{\sigma}$.

To obtain $D$ we repeat the above process a transfinite number of times exceeding the cardinality of $C$, taking at each limit step, the canonical full extension of the union of the previously constructed algebras. $D$ is the union of this new chain of extensions.

Q.E.D.

3. The case of equality algebras. Many results of $\$ 2$ can be stated for equality algebras. In these statements all subalgebras of a given algebra are assumed to have a common equality and all homomorphisms are assumed to be equality homomorphisms. In most cases these statements need not be given new proofs but can be deduced easily from the corresponding statements for general polyadic algebras as we shall show presently. For this we need a lemma.

LEMma 3.1. If $\left\{A_{h} \mid h \in H\right\}$ is a set of subalgebras of an algebra $C$, the union of which generates $C$, and if $E$ is a common equality for the $A_{h}$, then $E$ is an equality for $C$.

Proof. We know that $E$ is reflexive. We show that $E$ is substitutive, i.e., for all $p \in C$ and $i, j \in I$ 


$$
p \wedge E(i, j) \leqq S(i / j) p .
$$

Let $\Sigma$ be the set of elements $p$ of $C$ for which (1) holds; we show that $\Sigma=C$. It is easy to see that $\Sigma$ is closed under $\vee$ and $\Lambda$. Let $B_{0}$ be the Boolean algebra generated by the $A_{h}$. Since an element of $B_{0}$ is a union of intersections of elements in the $A_{h}$, we have that $B_{0} \subset \Sigma$. $B_{0}$ is obviously closed under all $S(\alpha), \alpha \in I^{I}$, and generates $C$ polyadically. Therefore, by the prenex normal form theorem (see [17]), an arbitrary element of $C$ is the result of applying a finite number of quantifiers $\exists(i)$ and $\forall(j)$ to an element of $B_{0}$. For each non-negative integer $n$, let $B_{n}$ be the set of elements of $C$ obtainable by prefixing at most $n$ quantifiers of the form $\exists(i)$ or $\forall(j)$ to an element of $B_{0}$. Each $B_{n}$ is a set (actually a Boolean algebra) closed under all $S(\alpha)$; this is easily verified by induction on $n$ using $\left(P_{6}\right)$ in the definition of polyadic algebras.

We will be finished if we can show that $B_{n} \subset \Sigma$ implies that $B_{n+1} \subset \Sigma$, for all $n$. Suppose therefore that $B_{n} \subset \Sigma$ and let $p \in B_{n}$. We have to show that $Q_{k} p \wedge E(i, j) \leqq S(i / j) Q_{k} p$, where $Q_{k}$ is either $\exists(k)$ or $\forall(k)$, for all $i, j, k \in I$. Let $k^{\prime} \in I$ be neither $i$ nor $j$ and let $p_{1}=S(\beta) p$ where $\beta$ is the transformation that interchanges $k$ and $k^{\prime}$ but leaves all other variables fixed. Since $p_{1} \in B_{n}$, we have (1) with $p_{1}$ instead of $p$. Applying $Q_{k}$, to both members we have, since a quantifier is monotone, $Q_{k^{\prime}} p_{1} \wedge E(i, j) \leqq S(i / j) Q_{k^{\prime}} p_{1}$. But $Q_{k^{\prime}} p_{1}=Q_{k} p$.

An application of Lemma 1.1 immediately yields the equality version of Corollary 2.3. For the equality versions of Proposition 2.4, Theorem 2.7 and Theorem 2.9 , apply Lemma 3.1 . Thus in 2.4 , simply replace $D$ by the subalgebra generated by all subalgebras $\rho^{i} C$ where $\rho$ is the automorphism extending $\sigma$ and $i$ runs through all integers; then apply Corollary 2.3. The easiest way to obtain the equality version of Theorem 2.11 is to go through the proof again and use this time the equality version of Proposition 2.4 where we used Proposition 2.4 before, noting also that the canonical full extension of a rich equality algebra $D^{*}$ has the same equality as $D^{*}$.

Theorem 2.6 does not work for equality algebras in general; in order to state the correct generalization of this theorem to equality algebras, we need some definitions. If $A$ is an algebra with equality $E$, we say that $A$ (or $E$ ) is of characteristic zero if for all finite set of variables $J$ we have

$$
\bigvee\{E(i, j) \mid i \neq j ; i, j \in J\} \neq 1 \text {. }
$$

If $A$ is not of characteristic 0 we say that it is of characteristic $n>0$ for the least integer $n$ for which (2) fails to hold for a set of variables $J$ containing $n+1$ elements.

The following lemma is well known.

Lemma 3.2. Let $A$ be a simple equality algebra and let $X$ be a set. Then, $A$ is of characteristic 0, a sufficient condition so that there exists an equality 
imbedding $A \rightarrow C_{X}$, is that $|X| \geqq|A|$; on the other hand, if $A$ is of nonzero characteristic $n$, such an imbedding exists iff $|X|=n$.

Proof. Assume $A$ of characteristic 0 and let $|X|=\alpha$. Let $K$ be a new set of cardinality $\alpha$ and fix the variables in $K$ of an $(I \cup K)$-dilation of $A$ to obtain an algebra $A(K)$. The set of closed elements of $A(K),\left\{E\left(k_{1}, k_{2}\right) \mid k_{1} \neq k_{2} ; k_{1}, k_{2} \in K\right\}$ generates a proper ideal. By dividing $A(K)$ by a maximal extension of this ideal we obtain a simple extension of $A$, the set $Y_{1}$, say, of all constants of which is of cardinality $\alpha$. Also $\left|A\left(Y_{1}\right)\right|=\alpha$ since $\alpha \geqq|A|$. If now $Y$ is the set of all constants of a rich simple extension $A(Y)$ of $A\left(Y_{1}\right)$, the canonical representation of $A(Y)$ yields an equality imbedding $A \rightarrow C_{Y}$. As we may suppose $|Y|=\alpha$, we may also suppose that $C_{Y}$ is isomorphic to $C_{X}$.

The rest of the lemma follows easily from the representation theorem for equality algebras and the easily established fact that any simple rich equality extension of a simple equality algebra of characteristic $n>0$ is bound to have exactly $n$ constants.

Q.E.D.

For the generalization of Theorem 2.6 to equality algebras we confine our attention to simple algebras.

THEOREM 3.3. Any family of simple equality algebras all having the same characteristic $n \geqq 0$ can be imbedded in one simple equality algebra of the same characteristic.

Proof. Let $\left\{A_{h} \mid h \in H\right\}$ be the given family. We use Lemma 3.2. If $n>0$, the common extension is $C_{X}$ with $|X|=n$. If $n=0$, we let $X$ be a set such that $|X| \geqq\left|A_{h}\right|$ for all $h \in H$, and again the common extension is $C_{X}$. Q.E.D.

Before we come to cardinality and unicity considerations concerning homogeneous extensions we study in the next section another problem related to automorphisms of polyadic algebras.

4. Normal extensions. By analogy with elementary field theory, we say that an extension $B$ of an algebra $A$ is normal if for every element $p$ in $B-A$ there exists an $A$-automorphism $\sigma$ of $B$ which moves $p$, i.e., $\sigma(q)=q$ for all $q \in A$ and $\sigma(p) \neq p$. This means that $A$ is the fixed subalgebra of $B$ for the group of all $A$-automorphisms of $B$, i.e., $A$ is equal to the subalgebra of $B$ formed of all elements of $B$ left invariant by all $A$-automorphisms of $B$.

The main result of this section asserts that, for simple algebras, any extensions can be imbedded into a rich homogeneous normal extension (Corollary 4.6). The preliminary results are valid also for nonsimple algebras. Our procedure makes use of a method of proof due to A. Robinson [38] and which can be summed up in the following

Lemma 4.1. Let $B$ and $B_{1}$ be two Boolean algebras such that $B \subset B_{1}$ and 
$p \in B_{1}-B$. Then, there is a maximal ideal $M$ of $B$ such that neither $p$ nor $p^{\prime}$ is in the ideal of $B_{1}$ generated by $M$.

Proof. Let $J(p)=\{q \mid q \in B$ and $q<p\}$ and $J\left(p^{\prime}\right)=\left\{q \mid q \in B\right.$ and $\left.q<p^{\prime}\right\}$; these are two ideals of $B$. Let $K=J(p) \cup J\left(p^{\prime}\right)$. $K$ generates a proper ideal, for $q_{1} \vee q_{2}=1$ with $q_{1}<p$ and $q_{2}<p^{\prime}$ is a contradiction. Let $J$ be any proper ideal of $B$ containing $K$. Then we show that $p \notin \bar{J}=\left\{q_{1} \mid q_{1} \in B_{1}\right.$ and $q_{1} \leqq q$ for some $q \in J\}$, the ideal of $B_{1}$ generated by $J$. Indeed, if $p<q$ with $q \in J(p=q$ is excluded since $p \notin B$ ) we would have $q^{\prime}<p^{\prime}$ and therefore $q^{\prime} \in K \subset J$, contradicting the fact that $q \in J$. Similarly $p^{\prime} \notin \bar{J}$. Let $M$ be a maximal $J$. Q.E.D.

Our next theorem will later be interpreted as an algebraic version of Beth's theorem in the theory of definition (Theorem 11.1).

THEOREM 4.2. Let $A_{1}$ be any extension of any algebra $A$ and $p \in A_{1}-A$. Then there exist two homomorphisms $f_{1}$ and $f_{2}$ of $A_{1}$ into some algebra $D$ such that $f_{1}(p) \neq f_{2}(p)$ and $f_{1}\left|A=f_{2}\right| A$.

Proof. Let $\left\{i_{1}, \cdots, i_{n}\right\}$ support $p$ and let $A_{1}\left(d_{1}, \cdots, d_{n}\right)$ be obtained by fixing $n$ new variables. We shall abbreviate $\left(d_{1}, \cdots, d_{n}\right)$ by $d$ and shall write $p(d)$ for $S\left(d_{1} / i_{1}\right) \cdots S\left(d_{n} / i_{n}\right) p$. Thus $p(d)$ is in the center $B_{1}$ of $A_{1}(d)$ but not in the center $B$ of $A(d)$. (This is the only property of $d$ used hereafter.) By Lemma 4.1, let $M$ be a maximal ideal of $B$ admitting two maximal extensions $M_{1}$ and $M_{2}$ in $B_{1}$ such that $p(d) \in M_{1}$ and $p(d)^{\prime} \in M_{2}$. Let $\left(h_{i}, \delta\right): A_{1}[d] \rightarrow A_{1}^{(i)}[d]$, be the natural homomorphism with kernel $M_{i}, i=1,2$. If $A^{(i)}[d], i=1,2$, are the images of $A[d]$ by these homomorphisms, we have an isomorphism $(\sigma, \delta): A^{(1)}[d] \rightarrow A^{(2)}[d]$ such that $h_{2}=\sigma h_{1}$ on $A(d)$. Let $\left(g_{i}, \bar{g}_{i}\right): A_{1}^{(1)}[d] \rightarrow C[X]$, where $C[X]$ is a suitable pair and $i=1,2$, be monomorphisms such that the diagram

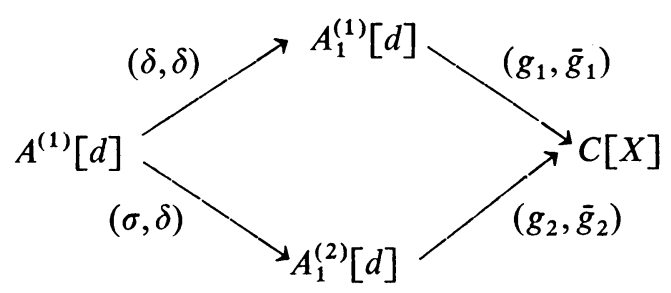

is commutative. This is possible by Theorem 2.7 when stated for pairs.

Set $D=C(X) ; f_{i}=g_{i} h_{i}$ for $i=1,2$; and $e_{j}=g_{1}\left(d_{j}\right)=\bar{g}_{2}\left(d_{j}\right)$ for $j=1, \cdots, n$. e have, for $q \in A, f_{1}(q)=g_{1} h_{1}(q)=g_{2} \sigma h_{1}(q)=g_{2} h_{2}(q)=f_{2}(q)$. Also $f_{1}(p) \neq f_{2}(p)$ since $S\left(e_{1} / i_{1}\right) \cdots S\left(e_{n} / i_{n}\right) f_{1}(p)=0$ and $S\left(e_{1} / i_{1}\right) \cdots S\left(e_{n} / i_{n}\right) f_{2}(p)=1 . \quad$ Q.E.D.

If $A$ and $A_{1}$ have a common equality, all references to pairs can be eliminated if we use the equality version of Theorem 2.7 and the unicity part of Lemma 1.3.

Proposition 4.3. Let $A_{1}$ be a simple extension of a simple algebra $A$ and let $p \in A_{1}-A$. Then there exists a simple extension $D$ of $A_{1}$ and an A-automor- 
phism $\rho$ of $D$ such that $|D|=\left|A_{1}\right|$ and $\rho(p) \neq p$. If $A$ and $A_{1}$ have a common equality then it may be assumed that $D$ shares this equality.

Proof. Let $f_{i}: A_{1} \rightarrow D_{1}, i=1,2$. be the monomorphisms given by Theorem 4.2. Apply Proposition 2.4 with $f_{i}\left(A_{1}\right)$ instead of $A_{i}(i=1,2), D_{1}$ instead of $C$ and $\sigma=f_{2} f_{1}^{-1}$. Obtain thus an extension $D_{2}$ of $D_{1}$ and an automorphism $\rho$ of $D_{2}$ extending $\sigma$, which means that $\rho f_{1}=f_{2}$. Identify $A_{1}$ with $f_{1}\left(A_{1}\right)$ say, and let $D$ be the smallest subalgebra of $D_{2}$ containing $f_{1}\left(A_{1}\right)$ and closed under $\rho$; this is the required extension. For $D$ is generated by $\bigcup_{i=-\infty}^{\infty} \rho^{i} f_{1}\left(A_{1}\right)$, a set of cardinality $\left|A_{1}\right|$, and hence $|D|=\left|A_{1}\right|$. If $A$ and $A_{1}$ have a common equality $E$, then $E$ being left fixed by $\rho, D$ also admits the equality $E$ by Lemma 3.1. Also, for $q \in A$, we have $\rho\left(f_{1} q\right)=f_{2}(q)=f_{1}(q)$ and $\rho\left(f_{1} p\right)=f_{2}(p) \neq f_{1}(p)$.

Q.E.D.

For any infinite cardinal number $\beta$ an algebra $C$ is said to be $\beta$-homogeneous if any monomorphism $\sigma: C_{1} \rightarrow C_{2}$ between subalgebras of $C$ of cardinality $<\beta$, extends to an automorphism of $C$. This concept is due to B. Jónsson [22]. If $X$ is an infinite set of cardinality $\alpha$ and $C$ is an homogeneous extension of $C_{X}$ then $C$ is $\alpha^{+}$-homogeneous. This follows from the fact that any algebra of cardinality $\leqq \alpha$ can be imbedded in $C_{X}$.

In what follows we let $X$ be an infinite set of cardinality $\alpha$ and $C_{X}^{*}$ be any simple $\alpha^{+}$-homogeneous extension of $C_{X}$.

Lemma 4.4. Let $A_{1}$ be a subalgebra of $C_{X}^{*}$ of cardinality $\leqq \alpha$. Then any extension $D$ of $A_{1}$ of cardinality $\leqq \alpha$ is equivalent to an extension of $A_{1}$ in $C_{X}^{*}$.

Proof. Let $\sigma: D \rightarrow C_{X}^{*}$ be any monomorphism and let $\rho$ be an automorphism of $C_{X}^{*}$ extending $\sigma \mid A_{1}$. Then if we set $\sigma_{1}=\rho^{-1} \sigma, \sigma_{1}$ is a monomorphism of $D$ into $C_{X}^{*}$ which leaves $A_{1}$ elementwise fixed.

Q.E.D.

THEOREM 4.5. $C_{X}^{*}$ is a normal (homogeneous) extension of any subalgebra $A$ of cardinality at most $\alpha$.

Proof. Let $p \in C_{X}^{*}-A$ and let $A_{1}$ be a subalgebra of $C_{X}^{*}$, of cardinality at most $\alpha$ and containing $A$ and $p$. Let, by Proposition $4.3, D$ be an extension of $A_{1}$ of cardinality at most $\alpha$, admitting an automorphism $\rho$ which moves $p$. By Lemma 4.4 we may assume that $D$ is also contained in $C_{X}^{*}$. The automorphism $\rho$ extends to an automorphism of $C_{X}^{*}$ because of the $\alpha^{+}$-homogeneity of $C_{X}^{*}$. Q.E.D.

The main result of this section is obtained as a corollary:

Corollary 4.6. Let $A_{1}$ be any simple extension of a simple algebra $A$. Then $A_{1}$ can be extended to a simple rich homogeneous and normal extension $B$ of $A\left({ }^{5}\right)$.

Proof. We let $\alpha=\left|A_{1}\right|$ and imbed $A_{1}$ into a rich $C_{X}^{*}$ which we choose as

(5) Corollary 2 of the author's abstract [5] is misstated: the statement becomes true if the word "simple" is deleted throughout. 
our $B$. This is indeed a rich simple homogeneous extension of $A$ since $|A| \leqq \alpha$. That $B$ is normal over $A$, is guaranteed by Theorem 4.5 .

Next we examine the case of equality algebras. Suppose that in Corollary 4.6, $A$ and $A_{1}$ have a common equality $E$; then we wish to show that $B$ can be chosen so that it shares this equality. For this, when $A_{1}$ is of characteristic 0 , it suffices to let the imbedding $A_{1} \rightarrow C_{X}^{*}$ of the above proof be an equality imbedding (Lemma 3.2). If $A_{1}$ is of characteristic $n$, it can be imbedded in $C_{X}$ with $|X|=n$. Since, then, $C_{X}$ has no proper simple equality extension, it is normal over any equality subalgebra $A$ by Proposition 4.3 , and it is an homogeneous extension of any such $A$ by the equality version of Theorem 2.11 .

Theorem 4.5 can be reformulated by saying that the function which assigns to any subalgebra $A$ of $C_{X}^{*}$ of cardinality at most $\alpha(=|X|, X$ an infinite set $)$ the group of $A$-automorphisms of $C_{X}^{*}$, is injective. No characterization of the groups of automorphisms of $C_{X}^{*}$ that are obtained in this manner will be discussed in this paper and, in any case, that cannot be attempted with any chance of success before some unicity statement is made regarding $C_{X}^{*}$. The situation is different for a finite set $X$ if we further confine ourselves to equality algebras; for, in this case, as we have seen, the role of $C_{X}^{*}$ is played simply by $C_{X}$. To any group $G$ of automorphisms of $C_{X}$ there corresponds a group $\tilde{G}=\{\tilde{\sigma} \mid \sigma \in G\}$ of permutations of $X$ and conversely (Theorem 1.4).

TheOREM 4.7. Let $X$ be a finite set. The correspondence which assigns to every equality subalgebra $A$ of $C_{X}$, the group of permutations of $X$ corresponding to the group of A-automorphisms of $C_{X}$ is an antiisomorphism of the lattice of all equality subalgebras of $C_{X}$ onto the lattice of all permutation groups of $X$.

Proof. The only nontrivial part of this statement there is still to prove is the ontoness. For this, it suffices to construct for any group $G$ of automorphisms of $C_{X}$ an element $p$ of $C_{X}$ such that for any automorphism $\sigma$ of $C_{X}, \sigma p=p$ iff $\sigma \in G$. Let $X=\left\{x_{1}, \cdots, x_{n}\right\}$. To construct $p$ we define an $n$-place predicate $R$ on $X$ by setting for any $\left(y_{1}, \cdots, y_{n}\right) \in X^{n}, R\left(y_{1}, \cdots, y_{n}\right)=1$ iff for some $\rho \in G, y_{i}=\tilde{\rho} x_{i}$; $i=1, \cdots, n$. If $\left\{i_{1}, \cdots, i_{n}\right\}$ is a set of distinct variables, it is easy to verify that $p=\hat{R}\left(i_{1}, \cdots, i_{n}\right)$ has the required property.

Q.E.D.

From the theorem and its proof it also follows that any equality subalgebra of $C_{X}$, for $X$ finite, can be generated by a single predicate in addition to the equality.

In Theorem 4.7 our development virtually makes contact with the Galois theory of M. Krasner [33-35]. When interpreted polyadically Krasner's theory establishes a one-one correspondence between all groups of permutations of an arbitrary set $X$ and all (not locally finite) complete (in the Boolean sense) equality subalgebras of the polyadic algebra $C$ of all functions (with or without finite support) from $X^{I}$ (with $|I| \geqq|X|$ ) to 0 . Another statement of Krasner's 
theory is that any isomorphism between two subalgebras of $C$ of the type just described can be extended to an automorphism of $C\left({ }^{6}\right)$.

It is possible to generalize Corollary 4.6 to nonsimple algebras using the results of $\$ 6$ (hence the continuum hypothesis) and the concept of free product of algebras with amalgamation of subalgebras as defined in [4].

5. Cardinality considerations. The main result of the previous section (Corollary 4.6) says nothing as to the "unicity" of the normal homogeneous extension $B$. Of course, any unicity statement about $B$ is bound to be conditional to some restriction on the cardinality of $B$. From our point of view, however, a best result would render $B$ unique by imposing on it some further algebraic condition that would entail such a cardinality restriction. Examples of what we have in mind are the algebraic closure of a field or the real closure of an ordered field. Unfortunately we have not been able to find such a result and the only known unicity statement (Theorem 6.3) will make the $\alpha^{+}$-homogeneous extension $C_{X}^{*}$ of $C_{X}$ unique $(|X|=\alpha \geqq \omega)$, and will be provided by a theorem of B. Jónsson on general algebraic systems. This theorem assigns a prominent role to cardinal numbers and its known proof has the disadvantage of requiring the generalized continuum hypothesis.

In this section we pave the way to the application of Jónsson's work to polyadic algebras and we prove that if in Corollary 4.6, $A_{1}$ is of cardinality $\alpha$, then $B$ can be chosen of cardinality at most $2^{\alpha}$. Indeed, as this is not much harder, we prove a theorem (Theorem 5.5) which comes as close to Theorem 6.3 (for the class $\mathscr{P}$ ) as we have been able to get without the continuum hypothesis. We shall also have occasion to invoke the results of this section in $\$ 12$ instead of Theorem 6.3, thus making $\$ 12$ also independent of the continuum hypothesis.

We begin by noting the rather superficial fact that in such considerations we may assume that $|I|=\omega$. This is a consequence of local finiteness. Indeed any algebra is a dilation of an algebra of degree $\omega$. It is easily shown that if $A$ and $B$ are any algebras and if $\sigma: A \rightarrow B$ is an homomorphism and $A^{+}$and $B^{+}$are dilations of $A$ and $B$ (with the same set of variables) then $\sigma$ extends uniquely to an homomorphism $\sigma^{+}: A^{+} \rightarrow B^{+}$; if $\sigma$ is a monomorphism (epimorphism) then $\sigma^{+}$ is also a monomorphism (epimorphism). If $B$ is a rich algebra, any dilation $B^{+}$ is also rich. Therefore if $B$ is a rich homogeneous normal extension of $A$ then $B^{+}$is also a rich homogeneous normal extension of $A^{+}$. If $A_{J}$ is a $J$-compression of $A$ with $|J|=\omega$ we call $\left|A_{J}\right|$ the effective cardinality $e$ of $A$. If we let $|I|=\beta$ and $|A|=\alpha$ then $\alpha=\beta \cdot e\left({ }^{7}\right)$. Hence if $B_{J}$ is the desired extension for $A_{J}$ with

(6) Although Krasner mentions only permutations of $I$ and not transformations in general, it can be shown that, in this context, any $S(\alpha)$ can be defined in terms of equality, arbitrary intersections and the $S(\beta), \beta$ ranging over the permutations of $I$.

(7) Unless $A$ is degenerate, i.e., consists only of closed elements, which, as $A$ is simple means that $A=0$. 
$\left|B_{J}\right| \leqq 2^{e}$ we have a similar extension $B$ for $A$ such that $|B| \leqq \beta 2^{e} \leqq 2^{\beta \cdot e}=2^{\alpha}$. In particular, as soon as $\beta \geqq 2^{e}$, we even have $\alpha=|A|=|B|=\beta$. For the rest of this chapter we shall therefore assume for the sake of simplicity that $|I|=\omega$. Our statements remain valid, if, in them, one interprets the cardinality of any algebra as the effective cardinality of that algebra.

Next we prove two easy lemmas concerning the cardinality of certain sets.

Lemma 5.1. If $|X|=\alpha \geqq \omega$, then $\left|C_{X}\right|=2^{\alpha}$.

Proof. There are $|I|$ finite subsets $J$ of $I$. For each such $J$ there are $2^{\alpha}$ elements of $C_{X}$ supported by $J$, for $X^{J}$ has $2^{\alpha}$ subsets. Therefore $\left|C_{X}\right|=|I| \cdot 2^{\alpha}=\omega \cdot 2^{\alpha}=2^{\alpha}$.

Q.E.D.

LEMMA 5.2. Let $\alpha$ be an infinite cardinal and $\mu$ be a type involving at most $\alpha$ relations. Then the number of isomorphism classes of relational systems of type $\mu$ and of cardinality at most $\alpha$ is $2^{\alpha}$.

Proof. For each (infinite) cardinal $\beta$ there are $2^{\beta}$ ways of choosing on $\beta$ a finitary relation and hence $\left(2^{\beta}\right)^{\alpha}$ ways of choosing $\alpha$ such relations. Assuming $\beta \leqq \alpha$ we have at most $2^{\alpha}$ isomorphism classes of systems of cardinality $\beta$. Therefore there are at most $\alpha \cdot 2^{\alpha}=2^{\alpha}$ isomorphism classes of systems of cardinality at most $\alpha$. That there are exactly $2^{\alpha}$ such systems can be seen, for instance, by observing that for each subset $\Lambda^{\prime}$ of the domain $\Lambda$ of $\mu$ there exists a system $\left\langle X, R_{\lambda}\right\rangle$ such that $|X|=\alpha$ and $R_{\lambda}=\varnothing$ iff $\lambda \in \Lambda^{\prime}$.

Q.E.D.

COROLLARY 5.3. The number of isomorphism classes of polyadic algebras of cardinality at most $\alpha$ is at most $2^{\alpha}$.

Proof. A polyadic algebra of degree $\omega$ can be considered as a relational system of a type involving $\omega$ relations: there is in addition to the relations standing for the Boolean operations of, say, "intersection" and "complementation," one (binary) relation for each quantifier $\exists(J)$ such that $J$ is finite, and one (binary) relation for each $S(\alpha)$ such that $\alpha$ is a finite transformation (see 7.6 in [13]). The result follows from Lemma 5.2.

Q.E.D.

The following theorem will enable us to cut down the cardinality of homogeneous extensions in general.

THEOREM 5.4. Let $\bar{D}$ be a rich extension of an algebra $C$ and let $G$ be a group of automorphisms of $\bar{D}$. Suppose that $\gamma=\omega|G|$ and $\theta=\omega|C|$. Then there exists a rich extension $D$ of $C$ contained in $\bar{D}$, invariant under $G$ and such that $|D| \leqq \gamma \theta$.

Proof. Let $B$ be the smallest subalgebra of $\bar{D}$, containing $C$ and closed under $G$; we have that $|B| \leqq \gamma \theta$ for $B$ is generated by $\bigcup\{\sigma C \mid \sigma \in G\}$, a set of cardinality at most $\gamma \theta$. Let $Z_{0}$ be a set of constants of $\bar{D}$ such that $B\left(Z_{0}\right)$ is rich and $\left|Z_{0}\right| \leqq \gamma \theta$. 
Let now $Z_{1}$ be the smallest set of constants of $\bar{D}$ containing $Z_{0}$ and invariant under $\bar{G}=\{\bar{\sigma} \mid \sigma \in G\}$; again $\left|Z_{1}\right| \leqq \gamma \theta$. By induction we construct an infinite sequence $\left\{Z_{i} \mid 0 \leqq i<\omega\right\}$ of sets of constants of $\bar{D}$ such that $\left|Z_{i}\right| \leqq \gamma \theta$ for all $i$; and, whenever $i$ is even, $B\left(Z_{i}\right)$ is rich; and, whenever $i$ is odd, $Z_{i}$ is invariant under $\bar{G}$ and hence $B\left(Z_{i}\right)$ is invariant under $G$. Letting now $Z=\bigcup_{i=0}^{\infty} Z_{i}$, the required algebra $D$ is $B(Z)$.

Q.E.D.

We can now state the main result of this section.

THEOREM 5.5. Let $\alpha$ be an infinite cardinal, $X$ a set such that $|X|=\alpha$, and $C$ a simple algebra of cardinality at most $2^{\alpha}$. Then there exists a simple rich $\alpha^{+}$-homogeneous extension $C_{X}^{*}$ of $C_{X}$ of cardinality $2^{\alpha}$ in which $C$ can be imbedded.

Proof. Let by Theorem 2.6, $C_{0}$ be a common extension of $C_{X}$ and $C$. By Lemma 5.1 we may assume that $\left|C_{0}\right|=2^{\alpha}$. Let $D$ be any simple rich homogeneous extension of $C_{0}$; we will obtain $C_{X}^{*}$ by a transfinite induction of length $2^{\alpha}$.

We choose, once for all, a set $S$ of subalgebras of $C$ made up of representatives of all isomorphism classes of algebras of cardinality at most $\alpha$; by Corollary 5.3, $|S| \leqq 2^{\alpha}$. If $C^{\prime}$ is any subalgebra of $D$ such that $\left|C^{\prime}\right| \leqq 2^{\alpha}$ and $\Sigma^{\prime}$ is the set of all monomorphisms from elements of $S$ into $C^{\prime}$ then $\left|\Sigma^{\prime}\right| \leqq 2^{\alpha}\left|C^{\prime}\right|^{\alpha}=2^{\alpha}$.

Assume we have constructed a chain $\left\{\left(C_{\rho}, G_{\rho}\right) \mid 0 \leqq \rho<2^{\alpha}\right\}$ such that

(i) $\left\{C_{\rho}\right\}$ is an increasing chain of rich subalgebras of $D$;

(ii) $\left\{G_{\rho}\right\}$ is an increasing chain of groups of automorphisms of $D$;

(iii) for each $\rho, C_{\rho}$ is invariant under $G_{\rho}$;

(iv) for each $\rho,\left|C_{\rho}\right|=\left|G_{\rho}\right|=2^{\alpha}$;

(v) for each $\rho$, if $\Sigma_{\rho}$ denotes the set of all monomorphisms of algebras in $S$ into $C_{\rho}$, any element of $\Sigma_{\rho}$ extends to (at least) one element of $G_{\rho+1}$. Let $C_{X}^{*}$ be the union of the $C_{\rho}$ and $G$ be the union of $G_{\rho}$; obviously, $C_{X}^{*}$ is a rich extension of $C_{0}$ in $D$ invariant under the group $G$ and $\left|C_{X}^{*}\right| \leqq 2^{\alpha}$. We show that $C_{X}^{*}$ is $\alpha^{+}$-homogeneous. Given an isomorphism $\sigma: A_{1} \rightarrow A_{2}$ between two subalgebras of $C_{X}^{*}$ of cardinality $\leqq \alpha$, we choose $\rho<2^{\alpha}$ and $A \in S$ such that $A_{1}$ and $A_{2}$ are contained in $C_{\rho}$ and there exists an isomorphism $\sigma_{1}: A \rightarrow A_{1}$. Set $\sigma_{2}=\sigma \sigma_{1}$. The monomorphisms $\sigma_{1}$ and $\sigma_{2}$ extend to automorphisms $\sigma_{1}^{*}$ and $\sigma_{2}^{*}$ in $G_{\rho+1} \subset G$, and hence to automorphisms of $C_{X}^{*}$. If we let $\sigma^{*}=\left(\sigma_{1}^{*}\right)^{-1} \sigma_{2}^{*}$, we obtain an automorphism in $G$ which extends $\sigma$.

To construct the chain $\left\{\left(C_{\rho}, G_{\rho}\right)\right\}$, we first let $G_{0}$ consist of the identity automorphism alone. Assuming $\left\{\left(C_{\rho^{\prime}}, G_{\rho^{\prime}}\right) \mid \rho^{\prime} \leqq \rho\right\}$ constructed with the above properties we let $G_{\rho+1}$ be generated by $G_{\rho}$ together with a set of automorphisms of $D$ obtained by extending each monomorphism in $\Sigma_{\rho}$ to some automorphism of $D$. Letting $C^{\prime}=C_{\rho}$ and $\Sigma^{\prime}=\Sigma_{\rho}$ above we have that $\left|\Sigma_{\rho}\right| \leqq 2^{\alpha}$ and since $\left|G_{\rho}\right|=2^{\alpha}$, we have $\left|G_{\rho+1}\right|=2^{\alpha}$. We then let $C_{\rho+1}$ be any rich extension of $C_{\rho}$ in $D$, invariant under $G_{\rho+1}$ and of cardinality $2^{\alpha}$ (Theorem 5.4). 
If $\rho<2_{\alpha}$ is a limit ordinal and $\left\{\left(C_{\rho^{\prime}}, G_{\rho^{\prime}}\right) \mid \rho^{\prime}<\rho\right\}$ has been defined we simply let $C_{\rho}$ and $G_{\rho}$ be the union of the $C_{\rho^{\prime}}$ and the union of the $G_{\rho^{\prime}}$ respectively. Q.E.D.

6. $\alpha^{+}$-universal-homogeneous algebras. Let $\mathscr{K}$ be a class of similar relational systems and $\beta$ an infinite cardinal number. A system $A$ in $\mathscr{K}$ is said to be $\beta$-homogeneous with respect to $\mathscr{K}$ if any isomorphism $\sigma: A_{1} \rightarrow A_{2}$ such that $A_{i} \subset A, A_{i} \in \mathscr{K}$ and $\left|A_{i}\right|<\beta$ for $i=1,2$, extends to an automorphism of $A$. A system $A$ in $\mathscr{K}$ is said to be $\beta$-universal (with respect to $\mathscr{K}$ ) if any system $B$ in $\mathscr{K}$ such that $|B| \leqq \beta$ can be imbedded in $A$. A system which is both $\beta$-universal and $\beta$-homogeneous will be said to be $\beta$-universal-homogeneous. These definitions are due to $\mathrm{B}$. Jónsson who has proved a general theorem as to the existence and unicity of such systems, under the assumption of the (generalized) continuum hypothesis. In this section we shall apply Jónsson's theorem, in conjunction with results of the previous sections, to several classes of polyadic algebras. For the whole of this section we assume the continuum hypothesis.

Jónsson's theorem can be stated as follows (see $[21 ; 22]$ ).

THEOREM 6.1. Let $\mathscr{K}$ be a class of similar relational systems of a type having at most $\omega$ relations. In order that, for each infinite cardinal $\alpha$, there exists one and, to within isomorphism, only one $\alpha^{+}$-universal-homogeneous system in $\mathscr{K}$ of cardinality $\alpha^{+}=2^{\alpha}$, it is sufficient that the following conditions be satisfied:

(I') for every cardinal $\beta$ there is $A \in \mathscr{K}$ such that $|A| \geqq \beta$;

(II) if $A_{1} \in \mathscr{K}$ and $A_{2}$ is isomorphic to $A_{1}$ then $A_{2} \in \mathscr{K}$;

(III) any two systems in $\mathscr{K}$ can be imbedded in a third system in $\mathscr{K}$;

(IV') if $f_{i}: A \rightarrow A_{i}, i=1,2$, are monomorphisms such that $A, A_{1}$, and $A_{2}$ are in $\mathscr{K}$, then there exist $B$ in $\mathscr{K}$ and monomorphisms $g_{i}: A_{i} \rightarrow B, i=1,2$, such that $g_{1} f_{1}=g_{2} f_{2}$;

(V) $\mathscr{K}$ is closed under unions of arbitrary chains;

(VI) for every infinite cardinal $\beta$, if $A \in \mathscr{K}$ and $B$ is a subsystem of $A$, such that $|B| \leqq \beta$, then there exists a subsystem $C$ of $A$ containing $B$ and such that $|C| \leqq \beta$ and $C \in \mathscr{K}$.

When $\mathscr{K}$ satisfies the conditions of Theorem 6.1 we shall denote by $U_{\alpha}(\mathscr{K})$ the unique $\alpha^{+}$-universal-homogeneous system in $\mathscr{K}, \alpha$ being any infinite cardinal. Before we apply Theorem 6.1 to polyadic algebras it is convenient to derive an easy corollary of it.

COROLlaRY 6.2. Let $\mathscr{K}$ and $\mathscr{K}^{\prime}$ be any two classes of similar relational systems both satisfying the conditions of Theorem 6.1 and such that $\mathscr{K}^{\prime} \subset \mathscr{K}$. Suppose moreover that every system in $\mathscr{K}$ can be imbedded in a system in $\mathscr{K}^{\prime}$ having the same cardinality. Then $U_{\alpha}(\mathscr{K})=U_{\alpha}\left(\mathscr{K}^{\prime}\right)$.

Proof. That $U_{\alpha}\left(\mathscr{K}^{\prime}\right)$ is $\alpha^{+}$-universal with respect to $\mathscr{K}$ is obvious from the 
hypothesis; we show that it is $\alpha^{+}$-homogeneous. Let $\sigma: A_{1} \rightarrow A_{2}$ be an isomorphism such that $A_{i} \in \mathscr{K},\left|A_{i}\right| \leqq \alpha$ and $A_{i} \subset U_{\alpha}\left(\mathscr{K}^{\prime}\right)$ for $i=1,2$. Let, for $i=1,2, A_{i}^{\prime}$ be an extension of $A_{i}$ such that $\left|A_{i}\right| \leqq \alpha$ and such that there exists an isomorphism $\sigma^{\prime}: A_{1}^{\prime} \rightarrow A_{2}^{\prime}$. By an argument like that of the proof of Lemma 4.4 with $U_{\alpha}\left(\mathscr{K}^{\prime}\right)$ in the role of $C_{X}^{*}$ we may assume that $A_{1}^{\prime}$ and $A_{2}^{\prime}$ are contained in $U_{\alpha}\left(\mathscr{K}^{\prime}\right)$. The $\alpha^{+}$-homogeneity of $U_{\alpha}\left(\mathscr{K}^{\prime}\right)$ yields an automorphism of $U_{\alpha}\left(\mathscr{K}^{\prime}\right)$ extending $\sigma^{\prime}$ and hence $\sigma$.

Q.E.D.

For the application of Jónsson's work to polyadic algebras we maintain the hypothesis $|I|=\omega$ so that, as pointed out earlier (proof of Corollary 5.3), polyadic algebras can be considered as relational systems of a type having $\omega$ relations. We now list some classes of polyadic algebras:

$\mathscr{P}$, the class of all algebras;

$\mathscr{S}$, the class of all simple algebras;

$\mathscr{R}$, the class of all rich algebras;

$\mathscr{E}$, the class of all equality algebras;

$\mathscr{E}_{n}, n \geqq 0$, the class of all equality algebras of characteristic $n$;

$\mathscr{S}_{n}=\mathscr{E}_{n} \cap \mathscr{S}, n \geqq 0$;

$\mathscr{R}_{n}=\mathscr{E}_{n} \cap \mathscr{R}, n \geqq 0$.

e note that in order to apply Jónsson's theorem to classes of equality algebras we must conceive an equality algebra as a relational system of a type different from that of polyadic algebras so as to make the concept of equality monomorphism a special case of the concept of monomorphism for relational systems in general. The type of equality algebras is obtained from that of polyadic algebras by adding to the relations standing for the Boolean operations and for $\exists(J)$ and $S(\alpha)$ (with $J$ and $\alpha$ finite) a distinguished element standing for $E(i, j)$ for some arbitrary but fixed pair of distinct variables $(i, j)$.

Now we can state the main result of this section which, with the help of the continuum hypotheses, supersedes some of our earlier results.

THEOREM 6.3. The classes $\mathscr{P}, \mathscr{R}, \mathscr{S}, \mathscr{S} \cap \mathscr{R}, \mathscr{S}_{0}$ and $\mathscr{S}_{0} \cap \mathscr{R}_{0}$ all satisfy the conditions of Theorem 6.1 and hence each of them admits, for any infinite cardinal $\alpha$, a unique $\alpha^{+}$-universal-homogeneous system.

Proof. Conditions II, V, and VI are trivial in all cases. Condition I' follows from Lemma 5.1, say, in all cases. Condition III follows from Theorem 2.6 for the first four cases, and from Theorem 3.3 for the remaining two cases. Condition IV' follows from Theorem 2.7 for the first four cases and from the equality version of this theorem for the last two cases.

Q.E.D.

We cannot apply Jónsson's theorem to the class $\mathscr{E}$, for III fails for that class. The same condition also fails for the classes $\mathscr{E}_{n}$ and $\mathscr{R}_{n}$ for $n \geqq 0$. If $n>0, \mathscr{S}_{n}$ does not satisfy I'. Also it is easy to show that a class of equality algebras, like $\mathscr{E}$ for instance, conceived as a class of polyadic algebras so that the concept of 
monomorphism for it is simply that of polyadic monomorphism, does not satisfy Condition V.

From Corollary 6.2 it follows immediately that the six $\alpha^{+}$-universal-homogeneous systems given by Theorem 6.3 are equal in pairs. More precisely we have

Corollary 6.4 .

$$
\begin{aligned}
U_{\alpha}(\mathscr{P}) & =U_{\alpha}(\mathscr{R}), \\
U_{\alpha}(\mathscr{S}) & =U_{\alpha}(\mathscr{S} \cap \mathscr{R}), \\
U_{\alpha}\left(\mathscr{S}_{0}\right) & =U_{\alpha}\left(\mathscr{S}_{0} \cap \mathscr{R}_{0}\right) .
\end{aligned}
$$

The last of these three systems has an equality, but we do not expect the first two to be equality algebras:

THEOREM 6.5. The algebras $U_{\alpha}(\mathscr{P})$ and $U_{\alpha}(\mathscr{S})$ have no equality.

Proof. Let $U$ be any of these two systems and assume that $U$ has an equality $E$. Let $A_{1}$ be any equality subalgebra of $U$ of cardinality $\leqq \alpha$. Applying Proposition 4.3 with $A=\{0,1\}$ and $p=E(i, j)$ for some pair of variables such that $i \neq j$, we obtain an extension $D$ of $A_{1}$ and an automorphism $\rho$ of $D$ such that $\rho(p) \neq p$. As in Lemma 4.4 we may assume that the extension $D$ of $A_{1}$ is contained in $U$. The automorphism $\rho$ extends to $U$. But $p$ is invariant under any automorphism of $U$. Therefore $U$ has no equality.

Q.E.D.

There is yet another class of systems to which we wish to apply Jónsson's theorem. Let $A$ be a simple equality algebra of characteristic 0 . We denote by $\mathscr{R}(A)$ the class of all simple rich extensions by addition of constants of $A$. We say that an algebra $A(X) \in \mathscr{R}(A)$ of cardinality $\beta$ is $\beta$-universal-homogeneous if any algebra $A(Y)$ of cardinality $\leqq \beta$ in $\mathscr{R}(A)$ can be imbedded in $A(X)$ by an $A$-monomorphism and if whenever $\sigma: A\left(X_{1}\right) \rightarrow A\left(X_{2}\right)$ is an $A$-isomorphism between two subextensions of $A(X)$ of cardinality less than $\beta, \sigma$ can be extended to an automorphism of $A(X)$. This concept becomes a special case of the general concept of $\beta$-universal-homogeneous systems of a type which, in addition to the relations standing for the polyadic structure, has one distinguished constant for each element of $A$. Note that this type may have more than $\omega$ relations; it has, indeed, exactly $|A|$ relations assuming $A \neq \mathbf{0}$.

THEOREM 6.6. For all cardinals $\alpha \geqq|A|, \mathscr{R}(A)$ admits one and to within A-equivalence, only one $\alpha^{+}$-universal-homogeneous system $U_{\alpha}[\mathscr{R}(A)]$. If $A$ is identified to a subalgebra of $U_{a}\left(\mathscr{S}_{0}\right)$ by an equality monomorphism and if $X$ is the set of all constants of $U_{\alpha}\left(\mathscr{S}_{0}\right)$ then $U_{\alpha}[\mathscr{R}(A)]=A(X)$.

Proof. It is obvious from Corollary 6.4 that $A(X)$ is an $\alpha^{+}$-universal-homogeneous system in $\mathscr{R}(A)$, since $A(X)$ is closed under any $A$-automorphism of $U_{a}\left(\mathscr{S}_{0}\right)$. Also it is easy to see as in the proof of Theorem 6.3 that $\mathscr{R}(A)$ satisfies 
the conditions of Theorem 6.1 except the basic one concerning the number of relations of the type of the systems in $\mathscr{R}(A)$ when $|A|>\omega$. The proof is complete for the case $|A|=\omega$ (the case where $|A|<\omega$ is the trivial one where $A=0$ ).

For the case where $|A|>\omega$ we note that Theorem 6.1 remains valid if in it we replace $\omega$ by any cardinal $\beta$ provided we restrict $\alpha$ by the condition $\alpha \geqq \beta$. Only trivial modifications to the proof of Theorem 6.1 in $[21 ; 22]$ are needed to establish this fact; indeed Lemma 2.8 in [21] is the only part of the proof that needs minor changes. However the reader need not verify the whole of this. For we have already proved the existence of $U_{\alpha}[\mathscr{R}(A)]$ above independently of this extension of Theorem 6.1, and for the unicity of this system it suffices to verify that the proof of the unicity part of Theorem 6.1, that is, Theorem B in [22], is independent of any hypothesis as to the number of relations of the type of the systems concerned.

Q.E.D.

\section{Chapter II. Representations of SIMPle fUll AlgEbras}

7. Strongly rich algebras. The aim of Chapter II is to obtain a description of all regular representations of $C_{X}$, i.e., of all monomorphisms of $C_{X}$ into regular functional algebras. Of course all $\mathbf{0}$-valued representations are of this type. As a first step in the solution of this problem, we study in this section the representations of certain algebras to be called strongly rich algebras. We begin by some general remarks concerning representations; we shall use the notations and results of $\S 1$.

Let $f$ be a (functional) representation of an algebra $A$, i.e., an homomorphism of $A$ into a functional algebra; if this functional algebra is $B$-valued and has domain $Y$, it will be found useful to denote the representation in full by $[f, A, Y, B]$. It is obvious, since every element $p$ of $A$ is in the range of some predicate $P$ of $A$, that $f$ is entirely determined once the $B$-valued predicates $(f P)^{*}$ of $Y$ are known, $P$ ranging over the set of all predicates of $A$, or even over some set of predicates generating $A$. Indeed if $P$ is $n$-ary and $p=P\left(i_{1}, \cdots, i_{n}\right)$, then for all $y \in Y^{I},(f p)(y)=(f P)^{*}\left(y_{i_{1}}, \cdots, y_{i_{n}}\right)$. Even though these are determined by the $(f P)^{*}$, we shall often, when dealing with a regular representation, write explicit formulae for the operations $(f Q)^{*}$ defined on $Y$ by virtue of Theorem $1.2, Q$ being an arbitrary operation of $A$.

Suppose $A$ has an equality $E$. A functional representation $[f, A, Y, B]$ is said to be reduced if $f E$ is a reduced equality. The reduction of a representation $[g, A, Z, B]$ is a reduced representation $[f, A, Y, B]$ such that $Y$ is the set of all equivalence classes of $Z$ under an equivalence relation " $\sim$ " defined by: $z_{1} \sim z_{2}$ for $z_{1}$ and $z_{2}$ in $Z$, if $(g E)^{*}\left(z_{1}, z_{2}\right)=1$. The representation $f$ is defined by the equation $(f p)(y)=(g p)(z)$ where $p \in A, y \in Y^{I}, z \in Z^{I}$; and, for all $i, z_{i}$ is in the equivalence class $y_{i}$. For further details, the reader is referred to $\S 6$ of [15].

A representation $[f, A, Y, B]$ is said to be irredundant if for all $b \in B$ there is a $p \in A$ and a $y \in Y^{I}$ such that $(f p)(y)=b$. An irredundant representation 
$[f, A, Y, B]$ can be obtained from any regular representation $\left[f, A, Y, B_{1}\right]$ by letting $B$ be the subalgebra of $B_{1}$ consisting of these elements $b$ which have the above property. What we shall obtain in this chapter is a description of the reduced, regular, irredundant representations of $C_{X}$.

Two representations $\left[f_{i}, A, Y_{i}, B_{i}\right], i=1,2$, of an algebra $A$ are equivalent if for some bijective map $\phi: Y_{1} \rightarrow Y_{2}$ and some isomorphism $\psi: B_{1} \rightarrow B_{2}$, we have, for all $p \in A$ and $y \in Y_{2}^{I}$

$$
\left(f_{2} p\right)(y)=\psi\left[\left(f_{1} p\right)\left(\phi^{-1} y\right)\right] .
$$

In case $B_{1}$ and $B_{2}$ are complete Boolean algebras, this definition simply means that there exists an isomorphism $\sigma: F\left(Y_{1}^{I}, B_{1}\right) \rightarrow F\left(Y_{2}^{I}, B_{2}\right)$ such that $\sigma f_{1}=f_{2}$. If $\left[f_{1}, A, Y_{1}, B_{1}\right]$ is a given representation, $\phi: Y_{1} \rightarrow Y_{2}$ is any bijective map, and $\psi: B_{1} \rightarrow B_{2}$ is any isomorphism, then (1) can be used to define a representation $\left[f_{2}, A, Y_{2}, B_{2}\right]$, equivalent to the given one. We say that $\psi$ and $\phi$ carry $f_{1}$ into $f_{2}$.

The (equivalence classes of) reduced, regular, and irredundant representations of a simple equality algebra $A$ correspond biuniquely to the (equivalence classes of) rich extensions of $A$ by addition of constants. The correspondence assigns to such an extension $A(Y)$, the restriction to $A$ of the canonical representation of $A(Y)$. Conversely if $[f, A, Y, B]$ is a reduced, regular, and irredundant representation, $(f A)(\hat{Y})$ becomes a rich extension of $A$ by addition of constants after identifying $A$ with $f A$.

We say that a representation $\left[g_{1}, A_{1}, Y_{1}, B_{1}\right]$ is a subrepresentation of a representation $\left[g_{2}, A_{2}, Y_{2}, B_{2}\right]$ if $Y_{1} \subset Y_{2}, A_{1} \subset A_{2}, B_{1} \subset B_{2}$ and for all $p \in A_{1}$ and $y \in Y_{1}^{I},\left(g_{1} p\right)(y)=\left(g_{2} p\right)(y)$. Equivalently we say that $g_{2}$ is an extension of $g_{1}$ or contains $g_{1}$. If $A_{1}$ is a rich equality subalgebra of a rich equality algebra $A_{2}$ and if $g_{1}$ and $g_{2}$ are their canonical representations respectively, then $g_{2}$ contains $g_{1}$.

Next we shall express an arbitrary extension by addition of constants $A(Y)$ of an (arbitrary) algebra $A$ as a quotient of a free extension $A(K)$ of $A$. For convenience we assume that $A$ has an equality. An extension by addition of constants $B$ of $A$ is said to be free if for some set of constants $K$ of $B$ for which $B=A(K)$, the following property holds; for any couple $\left(h_{1}, h_{2}\right)$ where $h_{1}$ is an (equality) homomorphism of $A$ into some algebra $C$ and $h_{2}$ is a mapping from $K$ into constants of $C$, there exists an homomorphism $h: A(K) \rightarrow C$ such that $h \mid A=h_{1}$ and $h \mid K=h_{2}, h$ being the mapping from constants of $A$ to constants of $C$ defined by $h$ (Lemma 1.3). The set of constants $K$ is said to be free on $A$. It is clear that the cardinality of $K$ determines $A(K)$ to within equivalence. To prove the existence of free extensions we let $A(K)$ be obtained by fixing the variables in $K$ of an $(I \cup K)$-dilation $A^{+}$of $A$ (we assume, of course, that $I \cap K=\varnothing)$. Assuming $C$ and $\left(h_{1}, h_{2}\right)$ given we proceed to define $h$. We let $p \in A(K)$ have support $\left\{i_{1}, \cdots, i_{m}, k_{1}, \cdots, k_{n}\right\}$ as an element of $A^{+}$. Identifying the predicates of $A$ with their extension to $A^{+} p$ has the form 
$P\left(i_{1}, \cdots, i_{m}, k_{1}, \cdots, k_{n}\right)$ for some $(m+n)$-predicate $P$ of $A$. We set $h p=\left(h_{1} P\right)\left(i_{1}, \cdots, i_{m}, h_{2} k_{1}, \cdots, h_{2} k_{n}\right)$. The verification that $h$ is an homomorphism with the required properties is straightforward and will be omitted.

To express an arbitrary extension by constants $A(Y)$ as a quotient of a free extension we let $A(K)$ be such that $|K| \geqq|Y|$ and we choose for $h_{1}$ the identity isomorphism and for $h_{2}$ an arbitrary surjective mapping $K \rightarrow Y$. If $N$ is the kernel of an homomorphism $h$ inducing $\left(h_{1}, h_{2}\right)$, we of course have that $A(Y)$ is equivalent to $A(K) / N$.

We may mention here without a proof a fact which will not be needed later but is perhaps of independent interest: if $A=C_{X}(X$ an infinite set) and $K$ is a singleton $\{k\}$ then there exists a set $K_{1}$ of constants of the free extension $A(k)$ which is free on $A$ and which has cardinality $|X|$.

We are now ready for a first easy lemma.

LEMMA 7.1. If $A$ is an equality algebra and $A(K)$ is a free extension then the set of all constants of $A(K)$ is the closure $\Gamma(K)$ of $K$ under the operations of $A$ (identified to their natural extension to $A(K)$ ).

Proof. Let $c$ be a constant of $A(K)$ and let $q=E(i, c)$. As an element of $A^{+}$, $q$ has support $\left\{i, k_{1}, \cdots, k_{n}\right\}$ say, with $\left(k_{1}, \cdots, k_{n}\right) \in K^{n}$ and is therefore of the form $P\left(i, k_{1}, \cdots, k_{n}\right)$ where $P$ is an $(n+1)$-place predicate of $A$ (identified to its extension to a predicate of $A^{+}$) single-valued in its last $n$ places. Let $T$ be the $n$-place operation of $A$ defined by $P$. We have $c=T\left(k_{1}, \cdots, k_{n}\right)$ Q.E.D.

To get any further we must now specialize to a particular class of polyadic algebras. We say that an algebra $A$ is strongly rich if for any element $p$ of $A$ with finite support $J \cup\{i\}$, there exists a $J$-term $t$ of $A$ such that $\exists(i) p=S(t / i) p$. Our next lemma asserts that this class contains that algebra in which we are especially interested.

Lemma 7.2. For any set $X, C_{X}$ is strongly rich.

Proof. The generic element of $C_{X}$ has the form $p=\hat{P}\left(i, i_{1}, \cdots, i_{n}\right)$ where $P$ is some $(n+1)$-place predicate of $X$. We look for an $n$-place operation $\hat{T}$ of $C_{X}$ such that

$$
\exists(i) \hat{P}\left(i, i_{1}, \cdots, i_{n}\right)=\hat{P}\left[\hat{T}\left(i_{1}, \cdots, i_{n}\right), i_{1}, \cdots, i_{n}\right] .
$$

This means that for all $\left(x_{1}, \cdots, x_{n}\right) \in X^{n}$,

$$
\bigvee\left\{P\left(x, x_{1}, \cdots, x_{n}\right) \mid x \in X\right\}=P\left[T\left(x_{1}, \cdots, x_{n}\right), x_{1}, \cdots, x_{n}\right] .
$$

Given $\left(x_{1}, \cdots, x_{n}\right)$, if the supremum in (2) is 1 , choose $x \in X$ such that $P\left(x, x_{1}, \cdots, x_{n}\right)$ $=1$ and set $T\left(x_{1}, \cdots, x_{n}\right)=x$. Otherwise, define $T\left(x_{1}, \cdots, x_{n}\right)$ arbitrarily. Q.E.D.

As a second example of strongly rich algebras we mention Peano algebras. Since this fact is not needed here, we omit the proof which is simple and is based on a well-known argument that can be found for instance in [40]. 
In order to obtain the canonical representation of an extension $A(Y)$ of a strongly rich equality algebra $A$ we prove some lemmas which will yield information regarding the set of all constants of such an extension.

LEMMA 7.3. If $A$ is a strongly rich algebra, so is every homomorphic image of $A$ and also every extension by addition of constants.

Proof. First let $h: A \rightarrow \bar{A}$ be an epimorphism. To show that $\bar{A}$ is strongly rich, let $p=h q \in \bar{A}$ where $q \in A$; and $q$, and hence also $p$, has finite support $J \cup\{i\}$. Let $t$ be a $J$-term of $A$ inducing a $J$-term $\bar{t}$ of $\bar{A}$ and such that $\exists(i) q=S(t / i) q$. Then, applying $h$ we get $\exists(i) p=S(\bar{t} / i) p$.

We turn now to the case of an extension by addition of constants $A(Y), Y$ being the set of all constants of $A(Y)$. Let $q$ be an element of $A(Y)$ supported by $J \cup\{i\}$ with $J=\left\{i_{1}, \cdots, i_{n}\right\}$. For some positive integer $m,\left(y_{1}, \cdots, y_{m}\right) \in Y^{m}$ and $(m+n+1)$-place predicate $P$ of $A$ we have $q=P\left(i, i_{1}, \cdots, i_{n}, y_{1}, \cdots, y_{m}\right)$. Since $A$ is strongly rich there exists an $(m+n)$-place operation $T$ of $A$ such that, for all $\left(j_{1}, \cdots, j_{m}\right) \in I^{m}$,

$$
\exists(i) P\left[i, i_{1}, \cdots, i_{n}, j_{1}, \cdots, j_{m}\right]=P\left[T\left(i_{1}, \cdots, i_{n}, j_{1}, \cdots, j_{m}\right), i_{1}, \cdots, i_{n}, j_{1}, \cdots, j_{m}\right] .
$$

Assuming that all variables in (3) are distinct and applying $S\left(y_{1} / j_{1}\right) \cdots S\left(y_{n} / j_{n}\right)$ we have $\exists(i) q=S\left[T\left(i_{1}, \cdots, i_{m}, y_{1}, \cdots, y_{m}\right) / i\right] q$.

Q.E.D.

LEMMA 7.4. Let $A$ be a rich equality algebra and $h: A \rightarrow A$ be an epimorphism. Then the mapping $h$ of the set of all constants of $A$ into that of $\bar{A}$ is surjective.

Proof. Let $d$ be a constant of $A$ and let $p \in A$ be such that $h p=E(i, d)$. Set $q=\exists(I-\{i\}) p$ so that $\{i\}$ supports $q$ and also $h q=E(i, d)$. Let $c$ be a constant of $A$ such that $S(c / i) q=\exists(i) q$. Applying $h$ we get $E[h(c), d]=1$ and hence $h(c)=d$.

Q.E.D.

It is now possible to give a description of the canonical representation of an arbitrary extension by addition of constants of a simple strongly rich equality algebra $A$. Such extensions are rich by virtue of Lemma 7.3. Let $Y$ be the set of all constants of such an extension; $A(Y)$ need not be simple. The description is dependent on some fixed epimorphism $A(K) \rightarrow A(Y), A(K)$ being a free extension. We let $N$ be the kernel of this homomorphism restricted to the center of $A(K)$; if $t$ is a constant of $A(K)$ we denote by $t / N$ the constant that $t$ induces on $A(Y)$ and we denote by the same letter an operation of $A$ and its extensions to $A(K)$ or to $A(Y)$. With these conventions it follows from (14) in $\S 1$ that, if $Q$ is an $n$-ary operation of $A$ and $t_{1}, \cdots, t_{n}$ are constants of $A(K)$,

$$
Q\left(t_{1}, \cdots, t_{n}\right) / N=Q\left(t_{1} / N, \cdots, t_{n} / N\right) .
$$

From Lemmas 7.1, 7.3 and 7.4 it follows immediately that $Y=\{t / N \mid t \in \Gamma(K)\}$. We shall denote this set by $\Gamma(K) / N$. We note that 


$$
t_{1} / N=t_{2} / N \text { iff } E\left(t_{1}, t_{2}\right) \in N^{\prime}
$$

for all $t_{1}$ and $t_{2}$ in $\Gamma(K), N^{\prime}$ being the filter of $A(K)$ corresponding to $N$.

For future reference we note the following consequence of the considerations just made.

LEMMA 7.5. Let $A$ be a strongly rich equality algebra and let $A(Y)$ be any extension by addition of constants. Then the set of all constants of $A(Y)$ is the closure of $Y$ under all operations of $A$.

The center of $A(K)$ is the Boolean algebra $\Pi(K)$ of elements of the form $P\left(k_{1}, \cdots, k_{n}\right)$ where $P$ is an $n$-place predicate of $A$ and $\left(k_{1}, \cdots, k_{n}\right) \in K^{n}$. Hence the center of $A(Y)$ is isomorphic to $\Pi(K) / N$. The residue class modulo $N$ of an element $b$ of $\Pi(K)$ is denoted by $b / N$.

The canonical representation of $A(Y)$ is seen to be equivalent to a representation $\left[f_{N}, A(Y), \Gamma(K) / N, \Pi(K) / N\right]$ defined by the equation

$$
\left(f_{N} P\right)^{*}\left(t_{1} / N, \cdots, t_{n} / N\right)=P\left(t_{1}, \cdots, t_{n}\right) / N,
$$

$P$ being an $n$-place predicate of $A$ and $t_{1}, \cdots, t_{n}$ being elements of $\Gamma(K)$. Any element $t$ of $\Gamma(K)$ has the form $T\left(k_{1}, \cdots, k_{m}\right)$ for some $m$-ary operation $T$ of $A$ and $\left(k_{1}, \cdots, k_{m}\right) \in K^{m}$. From (4) and (6) above and (10) in $\S 1$, it follows that for an $n$-ary operation $Q$ of $A$, we have

$$
\left(f_{N} Q\right) *\left(t_{1} / N, \cdots, t_{n} / N\right)=Q\left(t_{1}, \cdots, t_{n}\right) / N .
$$

Let $Z$ be the set of all constants of $A$. Any representation such as $f_{N}$ contains the canonical representation $(f, A, Z, 0)$ of $A$ (if we identify $z$ with $z / N$ for all $z \in Z$ ). The ideal $N$ of $A(K)$ is said to be flat if for all $k \in K$ there is a (necessarily unique) $z \in Z$ such that $E(z, k) \in N^{\prime}$. It is obvious that $f_{N} \mid A$ coincides with the canonical representation $f$ of $A$ if $N$ is flat.

Of course any ideal $N$ of any free extension $A(K)$ will yield a representation $f_{N}$ of $A$; it suffices to take $A(K) / N$ as $A(Y)$.

8. Generalized reduced powers. In this section we shall derive a description of all reduced, regular, irredundant representations of $C_{X}$ from that given in the preceding section for strongly rich algebras in general. This will be achieved by means of the concept of generalized reduced power of a set $X$ of which we immediately give the definition.

Let $X, W$ and $K$ be arbitrary sets. Denote by $F\left(W^{K}, X\right)$ the set of all functions $u$ from $W^{K}$ to $X$ with finite support (i.e., such that for some finite subset $L$ of $K, u\left(w_{1}\right)=u\left(w_{2}\right)$ for all elements $w_{1}$ and $w_{2}$ of $W^{K}$ for which $\left.w_{1}\left|L=w_{2}\right| L\right)$. Denote by $\mathscr{P}_{\omega}\left(W^{K}\right)$ the Boolean algebra of all subsets of $W^{K}$ whose characteristic functions have finite supports. Let $D$ be any filter of $\mathscr{P}_{\omega}\left(W^{K}\right)$. By the generalized reduced power $F\left(W^{K}, X\right) / D$ we understand the 
quotient set of $F\left(W^{K}, X\right)$ by the equivalence relation " $\sim$ " defined for elements $u_{1}$ and $u_{2}$ in $F\left(W^{K}, X\right)$ by

$$
u_{1} \sim u_{2} \text { if }\left\{w \mid w \in W^{K} \text { and } u_{1}(w)=u_{2}(w)\right\} \in D .
$$

That this is an equivalence relation follows easily from the fact that $D$ is a filter. The equivalence class of an element $u$ of $F\left(W^{K}, X\right)$ modulo this relation is denoted by $u / D$. If $D$ is an ultrafilter we shall say that $F\left(W^{K}, X\right) / D$ is a generalized ultrapower of $X$. When $K$ is a finite set the generalized reduced power $F\left(W^{K}, X\right) / D$ is just a reduced power $X^{H} / D$ with $H=W^{K}$, in the sense of $[8 ; 9]$; if $K$ is a singleton $\{k\}, W^{K}$ can be identified with $W$.

An arbitrary $n$-ary operation $T$ on $X$ induces on $F\left(W^{K}, X\right)$ an operation $T^{*}$ defined by

$$
\left[T^{*}\left(u_{1}, \cdots, u_{n}\right)\right](w)=T\left[u_{1}(w), \cdots, u_{n}(w)\right]
$$

for all elements $u_{1}, \cdots, u_{n}$ in $F\left(X^{K}, X\right)$ and $w \in W^{K}$. In particular, treating an element $x \in X$ as a unary function on $X$ identically equal to $x$, we let $x^{*}$ be the function in $F\left(W^{K}, X\right)$ identically equal to $x$.

The main result of this section is the following

THEOREM 8.1. Any reduced, regular, irredundant representation of $C_{X}$ is equivalent to a representation $\left[f_{D}, C_{X}, F\left(X^{K}, X\right) / D, \mathscr{P}_{\omega}\left(X^{K}\right) / D\right]$ defined by

$$
\left(f_{D} \hat{R}\right)^{*}\left(u_{1} / D, \cdots, u_{n} / D\right)=\left\{w \mid w \in X^{K} \text { and } R\left[u_{1}(w), \cdots, u_{n}(w)\right]=1\right\} / D
$$

where $R$ is an arbitrary $n$-place relation of $X$ and $u_{1}, \cdots, u_{n}$ are elements of $F\left(X^{K}, X\right)$. If $T$ is an $n$-ary operation of $X$ then

$$
\left(f_{D} \hat{T}\right)^{*}\left(u_{1} / D, \cdots, u_{n} / D\right)=T^{*}\left(u_{1}, \cdots, u_{n}\right) / D \text {. }
$$

In particular for any element $x$ of $X$

$$
\left(f_{D} \hat{x}\right)^{*}=x^{*} / D \text {. }
$$

Moreover we may assume that $|K|=\left|F\left(X^{K}, X\right) / D\right|$. Conversely any set $K$ and any filter $D$ of $\mathscr{P}_{\omega}\left(X^{K}\right)$ yield a representation defined by (3).

Proof. According to the results of $\S 7$, any representation of $C_{X}$ of the kind mentioned is equivalent to one of the form $\left[f_{N}, C_{X}, \Gamma(K) / N, \Pi(K) / N\right]$. To show that this representation is equivalent to that defined by (3) we define a bijective map $\phi: \Gamma(K) \rightarrow F\left(X^{K}, X\right)$ and an isomorphism $\psi: \Pi(K) \rightarrow \mathscr{P}_{\omega}\left(X^{\boldsymbol{K}}\right)$. The generic element of $\Gamma(K)$ has the form $\hat{T}\left(k_{1}, \cdots, k_{n}\right)$ where $T$ is an $n$-ary operation of $X$ and $\left(k_{1}, \cdots, k_{n}\right) \in K^{n}$. We set for all $x \in X^{K}$

$$
\phi\left[\hat{T}\left(k_{1}, \cdots, k_{n}\right)\right](x)=T\left(x_{k_{1}}, \cdots, x_{k_{n}}\right) .
$$

Similarly the generic element of $\Pi(K)$ has the form $\hat{P}\left(k_{1}, \cdots, k_{n}\right)$ where $P$ is an $n$-ary predicate of $X$ and $\left(k_{1}, \cdots, k_{n}\right) \in K_{n}$. We set by definition 


$$
\psi\left[\hat{P}\left(k_{1}, \cdots, k_{n}\right)\right]=\left\{x \mid x \in X^{K} \text { and } P\left(x_{k_{1}}, \cdots, x_{k_{n}}\right)=1\right\} .
$$

We verify that $\phi$ is bijective and $\psi$ is an isomorphism. To show that $\phi$ is surjective we let $u \in F\left(X^{K}, X\right)$ have support $\left\{k_{1}, \cdots, k_{n}\right\} ; u$ determines an $n$-ary operation $T$ on $X$ characterized by the equation $u(x)=T\left(x_{k_{1}}, \cdots, x_{k_{n}}\right)$ for all $x \in X^{K}$. Then $u=\phi\left[\hat{T}\left(k_{1}, \cdots, k_{n}\right)\right]$. The map $\phi$ is one-one: for let $t=\hat{T}\left(k_{1}, \cdots, k_{n}\right)$ and $t^{\prime}=\hat{T}^{\prime}\left(k_{1}^{\prime}, \cdots, k_{n^{\prime}}^{\prime}\right)$ be two distinct elements of $\Gamma(K)$, then $E\left(t, t^{\prime}\right) \neq 1$. We shift the scene of action from the set $L=\left\{k_{1}, \cdots, k_{n}, k_{1}^{\prime}, \cdots, k_{n^{\prime}}^{\prime}\right\}$ to a subset of $I$ by applying to this inequality a substitution $S(\sigma)$ of the $(I \cup K)$-dilation of $C_{X}$, $\sigma$ being a permutation of $I \cup K$ such that $\sigma L \subset I$. We obtain

$$
q=E\left[\hat{T}^{\prime}\left(\sigma k_{1}^{\prime}, \cdots, \sigma k_{n^{\prime}}^{\prime}\right), \hat{T}\left(\sigma k_{1}, \cdots, \sigma k_{n}\right)\right] \neq 1,
$$

with $q$ in $C_{X}$. Hence for some $y \in X^{I}, q(y)=0$. Now choosing $x \in X^{K}$ such that $x_{k}=y_{\sigma k}$ for all $k \in L$, we have that $(\phi t)(x) \neq\left(\phi t^{\prime}\right)(x)$, which means that $\phi(t) \neq \phi\left(t^{\prime}\right)$. That $\psi$ is an homomorphism is obvious; that its kernel is 0 can be shown again by shifting the scene of action from a finite subset of $K$ to one of $I$. To show that $\psi$ is surjective, we let $s$ be a function from $X^{K}$ to $\mathbf{0}$ with a finite support $\left\{k_{1}, \cdots, k_{n}\right\}$. This function $s$ determines an $n$-place predicate $P$ of $X$ characterized by the equation $s(x)=P\left(x_{k_{1}}, \cdots, x_{k_{n}}\right)$. Then we have indeed that $\psi\left[\hat{P}\left(k_{1}, \cdots, k_{n}\right)\right]$ is the set of which $s$ is the characteristic function.

Next we let $D=\psi\left(N^{\prime}\right)$. Of course, the isomorphism $\psi$ induces an isomorphism $\Psi: \Pi(K) / N \rightarrow \mathscr{P}_{\omega}\left(X^{K}\right) / D$. The mapping $\phi$ also induces a bijective mapping $\Phi: \Gamma(K) / N \rightarrow F\left(X^{K}, X\right) / D$; this is seen from (5) in $\S 7$ together with (1) and (7) above. Now we see from (6) of $\S 7$, that $\bar{\phi}$ and $\bar{\psi}$ carry $f_{N}$ into a representation $f_{D}$ defined by (3). (See the remarks following (1), §7.) Since $K$ and $N$ can be chosen arbitrarily, so can $D$.

To prove (4) we let $\hat{P}$ be the $(n+1)$-place predicate of $C_{X}$ corresponding to $\hat{T}$. By definition

(8) $(f \hat{P})^{*}\left(u / D, u_{1} / D, \cdots, u_{n} / D\right)=1$ iff $\left\{w \mid w \in X^{K}\right.$ and $\left.u(w)=T\left[u_{1}(w), \cdots, u_{n}(w)\right]\right\} \in D$.

If we let $u=T^{*}\left(u_{1}, \cdots, u_{n}\right)$, then the set in (8) is $X^{K} \in D$. Therefore by (10) in $\S 1$ we have (4).

Q.E.D.

Every representation $f_{D}$ contains a subrepresentation equivalent to the identity representation of $C_{X}$. For if for $x \in X$, we let $x^{+}=x^{*} / D$ we have from (1) that $x_{1}^{*} \neq x_{2}^{*}$ whenever $x_{1}$ and $x_{2}$ are distinct elements of $X$. From (3) it follows that

$$
\left(f_{D} \hat{R}\right)^{*}\left(x_{1}^{+}, \cdots, x_{n}^{+}\right)=R\left(x_{1}, \cdots, x_{n}\right)
$$

for all $n$-place predicates $R$ of $X$ and all $\left(x_{1}, \cdots, x_{n}\right) \in X^{n}$. If we let $X^{+}=\left\{x^{+} \mid x \in X\right\}$ we see that the bijective map $x \rightarrow x^{+}$carries the identity representation of $C_{X}$ onto a subrepresentation of $f_{D}$ with domain $X^{+}$.

Next we characterize the ultrafilters $D$ of $\mathscr{P}_{\omega}\left(X^{K}\right)$ such that $f_{D}$ is equivalent 
to the identity representation of $C_{X}$. For each $w \in X^{R}$, we let $D_{w}$, be the ultrafilter of all sets in $\mathscr{P}_{\omega}\left(X^{K}\right)$ containing $w$.

THEOREM 8.2. If $D$ is an ultrafilter of $\mathscr{P}_{\omega}\left(X^{K}\right)$, the representation $f_{D}$ of Theorem 8.1 is equivalent to the identity representation of $C_{X}$ iff $D$ is of the form $D_{w}$ for some $w \in X^{K}$.

Proof. Let the notation be as in the proof of Theorem 8.1. According to the results of the end of $\S 7, f_{N}$ is equivalent to the identity representation of $C_{X}$ iff $N$ is a flat ideal. So all we need to show is that $N$ is flat iff $\psi\left(N^{\prime}\right)=D_{w}$ for some $w \in X^{K}$.

Assume that $N$ is flat. Then for each $k \in K$ there is a unique $x^{(k)} \in X$ such that $\psi\left[E\left(\hat{x}^{(k)}, k\right)\right]=\left\{v \mid v \in X^{K}\right.$ and $\left.x^{(k)}=v_{k}\right\} \in D$. Let $w \in X^{K}$ be defined by $w_{k}=x^{(k)}$. We claim that $D=D_{w}$. It suffices to show that $D_{w} \supset D$. Let $\hat{P}\left(k_{1}, \cdots, k_{n}\right) \in N^{\prime}$; we have to show that $P\left(w_{k_{1}}, \cdots, w_{k_{n}}\right)=1$ which means that $P\left(x^{\left(k_{1}\right)}, \cdots, x^{\left(k_{n}\right)}\right)=1$. Since $E\left(\hat{x}^{\left(k_{i}\right)}, k_{i}\right) \in N^{\prime}$ for $i=1, \cdots, n$ we have $\hat{P}\left(\hat{x}^{\left(k_{1}\right)}, \cdots, \hat{x}^{\left(k_{n}\right)}\right) \in N^{\prime}$, but this element of $N^{\prime}$ can only be 0 or 1 since it is in $C_{X}$. Since $0 \notin N^{\prime}$ the element in question is 1 and this gives the desired conclusion.

Conversely we show that for any $w \in X^{R}$, the filter $N^{\prime}=\psi^{-1}\left(D_{w}\right)$ is flat. Indeed $N^{\prime}$ contains $E(\hat{x}, k)$ for all $x \in X$ and $k \in K$ such that $w_{k}=x$. Q.E.D.

So far we have been dealing only with generalized reduced powers $F\left(W^{K}, X\right) / D$ in which $W=X$. Of course in the representations in which such powers occur, the sets $W$ and $K$ can be replaced by any sets $W_{1}$ and $K_{1}$ having the same cardinality as $W$ and $K$ respectively. Our next theorem shows how the case where $|W| \neq|X|$ comes into the picture. For the sake of convenience we deal only with the case of generalized ultrapowers. This result will be needed for an application in $\$ 12$. We first state a lemma of which we omit the proof which is an easy exercise.

Lemma 8.3. Let $B$ be a simple rich algebra and $A$ be a subalgebra with equality and closed under all constants of $B$; then the canonical representation of $A$ is obtained (up to equivalence) by reducing the restriction to $A$ of the canonical representation of $B$.

THEOREM 8.4. Let $W$ be any set and $Y$ be the set of all constants of a simple extension $C_{W}(Y)$. Let $\left[h_{D}, C_{W}, F\left(W^{K}, W\right) / D, 0\right]$ be equivalent to the restriction to $C_{W}$ of the canonical representation of $C_{W}(Y)$. Then for any set $X$ such that $|X| \leqq|W|$ and any stretch imbedding $s: C_{X} \rightarrow C_{W}$, if $g$ is the canonical representation of $\left(s C_{X}\right)(Y)$, then the representation $g_{s}$ of $C_{X}$ is equivalent to a representation $\left[f_{D}, C_{X}, F\left(W^{K}, X\right) / D, 0\right]$ defined by (3) with $W^{K}$ instead of $X^{K}$. Moreover we may assume that $|K|=|Y|$.

Proof. That we may assume that $|K|=|Y|$ follows from Theorem 8.1. For the definition of "stretch imbedding" the reader is referred to the discussion following Corollary 2.5 . 
Let $\pi: W \rightarrow X$ be the surjective mapping inducing $s$. For all $n$-place predicates $R$ of $X,(s \hat{R})^{*}$ is an $n$-place predicate on $W$ defined by the equation

$$
(s \hat{R})^{*}\left(w_{1}, \cdots, w_{n}\right)=R\left(\pi w_{1}, \cdots, \pi w_{n}\right)
$$

for all $\left(w_{1}, \cdots, w_{n}\right) \in W^{n}$.

By Lemma 8.3 , the representation $g$ is equivalent to a representation having for domain the quotient of the set $F\left(W^{K}, W\right) / D$ by the equivalence relation " " defined by

$$
u_{1} / D \sim u_{2} / D \text { if }\left[h_{D}(s E)\right]^{*}\left(u_{1} / D, u_{2} / D\right)=1,
$$

where $E$ is the equality of $C_{X}$ and $u_{1}$ and $u_{2}$ are elements of $F\left(W^{K}, W\right)$. From (3) with $f_{D}=h_{D}, X=W$ and $\hat{R}=s E$ we have

$$
u_{1} / D \sim u_{2} / D \text { iff }\left\{w \mid w \in W^{K} \text { and }(s E)^{*}\left(u_{1} w, u_{2} w\right)=1\right\} \in D .
$$

From this and (10) with $\hat{R}=E$ we get

$$
u_{1} / D \sim u_{2} / D \text { iff }\left\{w \mid w \in W^{K} \text { and } \pi\left(u_{1} w\right)=\pi\left(u_{2} w\right)\right\} \in D .
$$

Now $\pi$ induces surjective maps $\tilde{\pi}: F\left(W^{K}, W\right) \rightarrow F\left(W^{K}, X\right)$ and $\pi_{*}: F\left(W^{K}, W\right) / D \rightarrow F\left(W^{K}, X\right) / D$. These are defined as follows: first for all $u \in F\left(W^{K}, W\right)$ and $w \in W^{K}$ we set $(\tilde{\pi} u)(w)=\pi(u w)$. That $\tilde{\pi}$ is surjective follows from the fact that $\pi$ is. Next we set for the same $u, \pi_{*}(u / D)=(\tilde{\pi} u) / D$. The verification that $\pi_{*}$ is well defined is straightforward and so is, from (13), the verification that

$$
\pi_{*}\left(u_{1} / D\right)=\pi_{*}\left(u_{2} / D\right) \text { iff } u_{1} / D \sim u_{2} / D
$$

for all $u_{1}$ and $u_{2}$ in $F\left(W^{K}, W\right)$.

From (14) it follows that $g \mid s C_{X}$ is equivalent to a representation $\left[g_{D}, s C_{X}, F\left(W^{K}, X\right) / D, 0\right]$ if we set for all $n$-place predicates $R$ of $X$ and elements $v_{1}, \cdots, v_{n}$ of $F\left(W^{K}, X\right)$

$$
\left[g_{D}(s \hat{R})\right]^{*}\left(v_{1} / D, \cdots, v_{n} / D\right)=\left[h_{D}(s \hat{R})\right]^{*}\left(u_{1} / D, \cdots, u_{n} / D\right),
$$

where $u_{i}$ is such that $v_{i}=\tilde{\pi} u_{i}$ for all $i=1, \cdots, n$. If finally, we set $f_{D}=g_{D} s$ we obtain a representation $\left[f_{D}, C_{X}, F\left(W^{K}, X\right) / D, 0\right]$ equivalent to gs and such that, for $R$ and $v_{1}, \cdots, v_{n}, u_{1}, \cdots, u_{n}$ as before

$$
\begin{aligned}
\left(f_{D} \hat{R}\right)^{*}\left(v_{1} / D, \cdots, v_{n} / D\right)=1 & \text { iff }\left[h_{D}(s \hat{R})\right]^{*}\left(u_{1} / D, \cdots, u_{n} / D\right)=1 \\
& \text { iff }\left\{w \mid w \in W^{K} \text { and }(s \hat{R})^{*}\left[u_{1}(w), \cdots, u_{n}(w)\right]=1\right\} \in D \\
& \text { iff }\left\{w \mid w \in W^{K} \text { and } R\left[\pi\left(u_{1} w\right), \cdots, \pi\left(u_{n} w\right)\right]=1\right\} \in D \\
& \text { iff }\left\{w \mid w \in W^{K} \text { and } R\left[v_{1}(w), \cdots, v_{n}(w)\right]=1\right\} \in D .
\end{aligned}
$$


9. A different approach. A natural question to ask is whether any generalized reduced power $F\left(W^{K}, X\right) / D$ yields a representation $f_{D}$ of $C_{X}$ if we define $f_{D}$ by (3) of $\S 8$ with $W^{K}$ instead of $X^{K}$. If $W=X$, Theorem 8.1 furnishes a positive answer to this question. If $|W| \geqq|X|$ a positive answer is obtained from Theorem 8.4 by using the stretch imbedding $s$ defined by any surjective map $\pi: W \rightarrow X$. But if $|W|<|X|$, even if $K$ is a singleton $\{k\}$ and we are dealing with a reduced power $X^{W} / D$, we do not seem to be able to answer the question with the techniques now at our disposal otherwise than by a direct check. To make the positive answer to the question follow from general principles, and to point the way to other possible generalizations of reduced powers, we now sketch an entirely different development which is somewhat closer to the model-theoretic treatment initiated by $\operatorname{Los}[37]$ and later pursued by various authors in [ $9 ; 29$; 32], for instance. Thus we show that reduced powers make their appearance in at least two essentially different ways in the theory of polyadic algebras.

First some notations. The direct product of a family of sets $\left\{Y_{m} \mid m \in M\right\}$ is denoted by $\prod Y_{m}$. If $Y=\prod Y_{m}$ and $y \in Y$, the $m$ th component of $y$ is denoted by $y^{m}$. If $y \in Y^{I}$, then $y^{m}$ is defined by the equation $y^{m}{ }_{i}=y_{i}^{m}$; either of these elements is also denoted by $y_{i}^{m}$. An element $y \in Y$ will sometimes be denoted by a symbol like $\left\{y^{m}\right\}$.

Let $\left\{\left[f_{m}, A, Y_{m}, B_{m}\right] \mid m \in M\right\}$ be a family of representations of a single algebra $A$. Setting $Y=\prod Y_{m}$ and $B=\prod B_{m}$, we define the direct product representation $[f, A, Y, B]$ by the equation

$$
(f p)(y)=\left\{\left(f_{m} p\right)\left(y^{m}\right)\right\} \text { for all } p \in A \text { and } y \in Y^{I} .
$$

That $f$ is indeed a representation is easily verified. If $A$ has an equality and all $f_{m}$ are reduced, $f$ is also reduced. A direct product of regular representations is also regular.

A regular representation $[f, A, Y, B]$ can be divided, by any filter $D$ of $B$ to yield a quotient representation $\left[f_{D}^{\prime}, A, Y, B / D\right]$ defined by $\left(f_{D}^{\prime} p\right)(y)=(f p)(y) / D$ for all $p \in A$ and $y \in Y^{I}$. For a proof of this, simply read "regular functional algebra", for "functionally rich functional algebra" in 17.2 of [13]. If $A$ has an equality $E$ and $f$ is reduced, $f_{D}^{\prime}$ is not reduced in general. In this case we define anew the quotient of $f$ by $D$ to be the reduction $\left[f_{D}, A, Y / D, B / D\right]$ of $f_{D}^{\prime}$. The domain $Y / D$ of this representation is the set of all equivalence classes $Y / D$ of elements of $Y$ under the equivalence relation " $\sim$ " defined by

$$
y_{1} \sim y_{2} \text { if }(f E)^{*}\left(y_{1}, y_{2}\right)=1 \text { for all } y_{1} \text { and } y_{2} \text { in } Y \text {. }
$$

If $P$ is any $n$-place predicate of $A$ and $\left(y_{1}, \cdots, y_{n}\right) \in Y^{n}$,

$$
\left(f_{D} P\right)^{*}\left(y_{1} / D, \cdots, y_{n} / D\right)=(f P)^{*}\left(y_{1}, \cdots, y_{n}\right) / D \text {. }
$$

Thus reduced regular representations of an equality algebra $A$ can be multiplied and divided to yield new reduced regular representations. Let, for instance, 
$\left\{\left[f_{m}, A, Y_{m}, \mathbf{0}\right] \mid m \in M\right\}$ be a family of reduced $\mathbf{0}$-valued representations of $A$. Identifying $\mathbf{0}^{M}$ with $\mathscr{P}(M)$, the Boolean algebra of all subsets of $M$, and dividing the direct product representation by an arbitrary filter $D$ of $\mathscr{P}(M)$ we obtain the representation $\left[f_{D}, A, \prod X_{m} / D, \mathscr{P}(M) / D\right]$ where for $x$ and $y$ in $\prod X_{m}$,

$$
x / D=y / D \text { iff }\left\{m \mid x^{m}=y^{m}\right\} \in D,
$$

and for any $n$-place predicate $P$ of $A$ and $\left(x_{1}, \cdots, x_{n}\right) \in\left(\prod X_{m}\right)^{n}$,

$$
\left(f_{D} P\right)^{*}\left(x_{1} / D, \cdots, x_{n} / D\right)=\left\{m \mid\left(f_{m} P\right)^{*}\left(x_{1}^{m}, \cdots, x_{n}^{m}\right)=1\right\} / D .
$$

The set $\prod X_{m} / D$ is known as a reduced product of the sets $X_{m}$. If $D$ is an ultrafilter, the same set is known as a prime reduced product or an ultraproduct $\left(^{8}\right)$.

As in the proof of Theorem 8.1 it can be shown that for an $n$-place operation $Q$ of $A$ and elements $x_{1}, \cdots, x_{n}$ of $\prod X_{m}$

$$
\left(f_{D} Q\right)^{*}\left(x_{1} / D, \cdots, x_{n} / D\right)=Q^{*}\left(x_{1}, \cdots, x_{n}\right) / D,
$$

where $Q^{*}$ is the direct product of the operations $\left(f_{m} Q\right)^{*}$ on the $X_{m}$.

In particular, if $\left[f, C_{X}, X, 0\right]$ is the identity representation, $M$ is any set and $D_{1}$ is any filter in $\mathscr{P}(M)$, the quotient by $D_{1}$ of the $M$ th power of $f$ is a representation $\left[f_{D_{1}}, C_{X}, X^{M} / D_{1}, \mathscr{P}(M) / D_{1}\right]$.

If $\left[f_{D}, C_{X}, F\left(W^{K}, X\right) / D, \mathscr{P}_{\omega}\left(W^{K}\right) / D\right]$ is a representation as in Theorem 8.1 , we see, by setting $M=W^{K}$ and by choosing for $D_{1}$ the filter generated in $\mathscr{P}(M)$ by $D$, that $f_{D}$ is a subrepresentation of $f_{D_{1}}$.

Let $[f, A, Y, B]$ be any (regular) representation and $Z$ an arbitrary subset of $Y$.

e say that $Z$ yields a (regular) subrepresentation of $Y$ if we obtain a (regular) representation $[g, A, Z, B]$ by setting for $p \in A$ and $z \in Z^{I},(g p)(z)=(f p)(z)$. Suppose $A$ has an equality and $f$ is reduced and regular. Then $Z$ yields a regular subrepresentation iff $(f A)(\hat{Z})$ is rich and $\hat{Z}$ is the set of all constants of $(f A)(\hat{Z})$. If, furthermore, $A$ is strongly rich, this is equivalent to saying that $Z$ is closed under all operations $(f Q)^{*}$ of $Y, Q$ being an arbitrary operation of $A$ (Lemma 7.5).

This result enables us to answer affirmatively the question raised at the beginning of this section. It suffices to verify that for any operation $T$ on $X$, $F\left(W^{K}, X\right)$ is closed under the direct power operation $T^{\#}$ on $X^{M}$ with $M=W^{K}$.

Any kind of subsets of $X^{M} / D$ of the form $U / D$ where $U$ is a subset of $X^{M}$ closed under all $T$ and $U / D=\{u|D| u \in U\}$ yields a subrepresentation of $\left[f_{D}, C_{X}, X^{M} / D, \mathscr{P}(M) / D\right]$ and a generalization of the concept of reduced power. For instance, Keisler's limit ultrapowers [23] are easily seen to be of this form. Many variations on this theme become possible: assuming again $M=W^{K}$ we may take for $U$ the set $F_{\alpha}\left(W^{K}, X\right)$ of all functions from $W^{K}$ to $X$ with a support of

(8) Note however that, unless $D$ is an ultrafilter, $\left(f_{D} \mathrm{P}\right)^{*}$ is not a $\mathbf{0}$-valued predicate of $\Pi X_{m} / D$. This is not in accord with the definition of reduced products of relational systems given in $[8 ; 9]$. 
cardinality less than $\alpha, \alpha$ being an arbitrary cardinal. If $\alpha=\omega$, this is $F\left(W^{K}, X\right)$, whereas if $\alpha>|K|$, this is $X^{M}$. Or we may take for $U$ the set of all elements of $X^{M}$ with a finite range. And so on.

\section{Chapter III. MODEL-THEORETIC INTER PRETATION}

10. Basic concepts. In this chapter we shall derive some model-theoretic results from the polyadic results obtained in the first two chapters. This section is devoted to defining the concepts from Model theory which we shall need and to describing the fundamental relationships between these and the theory of polyadic algebras.

The concepts of a type $\mu$ and of relational system of type $\mu$ have been defined in Chapter 0 . Let $\mu: \Lambda \rightarrow \omega$ be a type and $\left\{P_{\lambda} \mid \lambda \in \Lambda\right\}$ be a family of predicates of a polyadic algebra; the family is said to be of type $\mu$ if for all $\lambda, P_{\lambda}$ is a $\mu(\lambda)$ place predicate. By the polyadic algebra of a system $\left\langle X, R_{\lambda}\right\rangle$ we mean the polyadic algebra generated in $C_{X}$ by the predicates $\hat{R}_{\lambda}$ and the functional equality.

Two similar systems $\left\langle X, R_{\lambda}\right\rangle$ and $\left\langle Y, S_{\lambda}\right\rangle$ are said to be elementarily equivalent if there is a (necessarily unique) isomorphism $\phi$ of the polyadic algebra of the rst system onto that of the second system such that, for all $\lambda, \phi \hat{R}_{\lambda}=\hat{S}_{\lambda}\left({ }^{9}\right)$.

To every type $\mu$ is associated a first order predicate language $L(\mu)$ with identity $=$, and a $\mu(\lambda)$-place predicate symbol $F_{\lambda}$ for each $\lambda \in \Lambda$. The set of individual variables of $L(\mu)$ is taken to be $I$. The symbols $\vee, \wedge, \equiv,^{\prime}, \exists, \forall$ are used in the customary way as propositional connectives and quantifiers; it will be seen that this use of these symbols is compatible with their use in the theory of Boolean and polyadic algebras. We assume that the reader knows the meaning of such words as formula, free and bound variables, and statement or closed formula. We also assume that the reader knows what it means for a formula of $L(\mu)$ to be satisfied in a system $\left\langle X, R_{\lambda}\right\rangle$ of type $\mu$ for a particular assignment of values in $X$ to the variables, i.e., for a particular element $x$ of $X^{I}\left({ }^{0}\right)$. A formula is said to be valid in $\left\langle X, R_{\lambda}\right\rangle$ if it is satisfied in $\left\langle X, R_{\lambda}\right\rangle$ for all elements $x \in X^{I}$.

Added in proof. A model of a set of formulas $\Sigma$ is a system in which all members of $\Sigma$ are valid. A formula $A$ is a consequence of $\Sigma$ if $A$ is valid in all models of $\Sigma$. A theory is a set of formulas containing all its consequences. The set $\tilde{\Sigma}$ of all consequences of $\Sigma$ is a theory. A set $\Sigma$ is consistent if it has a model. A theory is complete if it is consistent and if, for every statement $H$, it contains either $H$ or $H^{\prime}$. The set of all formulas valid in a given system is a complete theory called the complete theory of that system.

It is known that the concept of consequence can also be defined by means of a finitary concept of deduction but this will not be needed here.

(9) This concept is independent of $I$ (as long as $I$ is infinite). Indeed it is easily shown that, if $A_{I}$ and $A_{I}+$ are the polyadic algebras of a relational system with respect to two sets of variables $I$ and $I^{+}$such that $I \subset I^{+}$, then $A_{I}+$ is the $I^{+}$-dilation of $A_{I}$. For a clue to the proof of this, see Theorem 4.4 in [7]. Then the assertion follows from remarks in $\$ 5$.

(10) For more details on this, see for instance [44]. 
We denote by $F(\mu)$ the set of all formulae of $L(\mu)$. Propositional connectives such as $\vee, \wedge$ and $\equiv$ act as binary operations on $F(\mu)$, and for each $i \in I, \exists(i)$ and $\forall(i)$ act as unary operations on the same set. By the polyadic algebra of a (consistent) theory $T$ we mean an algebra to be denoted by $F(\mu) / T$ and whose elements are the equivalence classes of $F(\mu)$ under an equivalence relation defined by: $H_{1} \sim H_{2}$ iff $H_{1} \equiv H_{2} \in T$ for all $H_{1}$ and $H_{2}$ in $F(\mu)$. For any formula $H$ we denote by $H / T$ the equivalence class of $H$. For any $\lambda$, there is a $\mu(\lambda)$-place predicate $F_{\lambda} / T$ of $F(\mu) / T$ defined by

$$
\left(F_{\lambda} / T\right)\left(i_{1}, \cdots, i_{\mu(\lambda)}\right)=F_{\lambda}\left(i_{1}, \cdots, i_{\mu(\lambda)}\right) / T .
$$

This set of equivalence classes becomes an equality polyadic algebra under Boolean operations and quantifiers which are induced by the corresponding propositional connectives and quantifiers as operations in $F(\mu)$. The substitution operator $S(\alpha)$ for $\alpha \in I^{I}$ is induced by the unary operation on $F(\mu)$ that consists in actually performing the transformation $\alpha$ on the free variables of formulae. The unit element of that algebra is the equivalence class $T$. The verification of these facts is a straightforward exercise based on the definition of the notion of consequence that we have given above. For further reading on the subject of the correspondence between first-order theories and polyadic algebras the reader is referred to the articles $[11 ; 19 ; 20 ; 36 ; 41 ; 43]\left({ }^{11}\right)$. As a Boolean algebra, the algebra $F(\mu) / T$ is known as the Tarski-Lindenbaum algebra of the theory $T$.

e next state a theorem that shows the simple connection between models of a theory $T$ and $\mathbf{0}$-valued representations of the algebra $F(\mu) / T$.

THEOREM 10.1. Let $T$ be any theory. If $\left\langle X, R_{\lambda}\right\rangle$ is a model of $T$, then there exists a reduced representation $[f, F(\mu) / T, X, 0]$ such that $\left(f F_{\lambda} / T\right)^{*}=R_{\lambda}$ for all $\lambda$. Conversely if $f$ is any reduced 0-valued representation then $\left\langle X,\left(f F_{\lambda} / T\right)^{*}\right\rangle$ is a model of $T$.

Proof. Given the model $\left\langle X, R_{\lambda}\right\rangle$ of $T$, the representation $f$ is defined as follows. or $H / T \in F(\mu) / T$ and $x \in X^{I},(f H / T)(x)=1$ iff $H$ is satisfied if we assign the value $x_{i}$ to the variable $i$ and $R_{\lambda}$ to $F_{\lambda}$ for all $\lambda$ and $i$. We omit the verifications. The converse part follows from the fact that for $H \in T, H / T=1$ and hence for any representation $f, H$ is valid in $\left\langle X,\left(f F_{\lambda} / T\right)^{*}\right\rangle$.

Q.E.D.

In particular, if $T$ is the complete theory of a system $\left\langle X, R_{\lambda}\right\rangle$ we obtain as a corollary the relationship between the polyadic algebra of the system and that of the theory.

Corollary 10.2. Let $T$ be the complete theory of a system $\left\langle X, R_{\lambda}\right\rangle$ and $A$ be the polyadic algebra of this system. Then there exists an isomorphism $f: F(\mu) / T \rightarrow A$ such that for all $\lambda, f F_{\lambda} / T=\hat{R}_{\lambda}$.

(11) Although some of these references deal mostly with cylindrical algebras, the reader will have no difficulty in adapting their contents to polyadic algebras using the results of [10]. 
A consequence of this is the connection between the concept of elementary equivalence as we have defined it and its more customary definition.

Corollary 10.3. Let $A_{1}$ and $A_{2}$ be the polyadic algebras of similar systems $\left\langle X_{1}, R_{\lambda}\right\rangle$ and $\left\langle X_{2}, S_{\lambda}\right\rangle$ respectively. Then the systems $X_{1}$ and $X_{2}$ have the same complete theory iff there is an isomorphism $\phi: A_{1} \rightarrow A_{2}$ such that $\phi\left(\hat{R}_{\lambda}\right)=\hat{S}_{\lambda}$ for all $\lambda$.

Let $\left\langle X, R_{\lambda}\right\rangle$ and $\left\langle Y, S_{\lambda}\right\rangle$ be relational systems of type $\mu$ and $\phi: X \rightarrow Y$ be a monomorphism. Assume, for convenience, that $\Lambda \cap X=\varnothing$. The imbedding $\phi$ is said to be elementary if the systems $\left\langle X,\left\{R_{\lambda} \mid \lambda \in \Lambda \cup X\right\}\right\rangle$ and $\left\langle Y,\left\{R_{\lambda} \mid \lambda \in \Lambda \cup X\right\}\right\rangle$ defined as follows are elementarily equivalent. The type $v$ of these systems is defined by $v \mid \Lambda=\mu$ and $v(\lambda)=1$ for all $\lambda \in X$. Also for $\lambda$ and $x \in X$ we have by definition $R_{\lambda}(x)=1$ iff $x=\lambda$ and for $y \in Y, S_{\lambda}(y)=1$ iff $y=\phi(\lambda)$. We see that type $v$ is obtained from type $\mu$ by adding one distinguished element for every element of $X$. If $\left\langle X, R_{\lambda}\right\rangle$ is a subsystem of $\left\langle Y, S_{\lambda}\right\rangle$ and the identity map $X \rightarrow Y$ is elementary then $Y$ is said to be an elementary extension of $X$, and $X$ an elementary subsystem of $Y$.

To see what this means in terms of polyadic algebras we first note that for $x \in X$ and $i \in I, \hat{R}_{x}(i)=E_{1}(i, \hat{x}), E_{1}$ being the equality of $C_{X}$. Similarly, if $E_{2}$ is the equality of $C_{Y}$ and $y=\phi x$, then $\hat{S}_{x}(i)=E_{2}(i, \hat{y})$. It follows that, if $A_{1}$ and $A_{2}$ are the polyadic algebras of the systems $\left\langle X, R_{\lambda}\right\rangle$ and $\left\langle Y, S_{\lambda}\right\rangle$ of type $\mu$; then the polyadic algebras of the corresponding systems of type $v$ are $A_{1}(\hat{X})$ and $A_{2}\left(\phi X^{\wedge}\right)$ respectively. From these remarks, the following theorem is obvious.

THEOREM 10.4. Let $\left\langle X, R_{\lambda}\right\rangle$ and $\left\langle Y, S_{\lambda}\right\rangle$ be systems of type $\mu$ with polyadic algebras $A_{1}$ and $A_{2}$ respectively. Then a monomorphism $\phi: X \rightarrow Y$ is elementary iff there exists a monomorphism $\phi_{*}: A_{1}(\hat{X}) \rightarrow A_{2}(\hat{Y})$ such that

(i) $\phi_{*} \hat{R}_{\lambda}=\hat{S}_{\lambda}$ for all $\lambda$; and

(ii) $\hat{y}=\phi_{*} \hat{x}$ iff $y=\phi x$ for all $x \in X$ and $y \in Y$.

Therefore if $\phi_{*}: A_{1}(\hat{X}) \rightarrow A_{2}(\hat{Y})$ is any monomorphism satisfying (i), then an elementary imbedding $\phi: X \rightarrow Y$ can be defined by (ii). Thus the concept of the polyadic algebra of a relational system enables us to reduce the concept of elementary monomorphism to that of monomorphism.

Let $\left\langle X, R_{\lambda}\right\rangle$ be a system with algebra $A$ and let $[f, A(\hat{X}), Y, 0]$ be a reduced representation. Then, if we set by definition, $R_{\lambda}^{+}=\left(f \hat{R}_{\lambda}\right)^{*}$ and $x^{+}=(f \hat{x})^{*}$, we obtain an elementary imbedding $\phi$ from $\left\langle X, R_{\lambda}\right\rangle$ into $\left\langle Y, R_{\lambda}^{+}\right\rangle$defined by $\phi(x)=x^{+}$for all $x \in X$. In particular, if $f$ is the restriction to $A(\hat{X})$ of a representation $f_{D}$ of $C_{X}$ with a generalized ultrapower $F\left(W^{K}, X\right) / D$ as domain, we have an elementary imbedding of $\left\langle X, R_{\lambda}\right\rangle$ into a system $\left\langle F\left(W^{K}, X\right) / D, R_{\lambda}^{+}\right\rangle$called a generalized ultrapower of the system $\left\langle X, R_{\lambda}\right\rangle$. If $R$ is any of the $R_{\lambda}$ with $\mu(\lambda)=n$ and if $u_{1}, \cdots, u_{n}$ are elements of $F\left(W^{K}, X\right)$, then 


$$
R^{+}\left(u_{1} / D, \cdots, u_{n} / D\right)=1 \text { iff }\left\{w \mid w \in W^{K} \text { and } R\left(u_{1}, \cdots, u_{n}\right)=1\right\} \in D .
$$

11. Theorems of Beth and Svenonius. In this section we shall use results of $\$ 4$ to obtain new proofs of two known results in the theory of definition. The first of these results is due to Beth and has already been given several proofs by different authors (see $[1 ; 38 ; 3 ; 32])$. The second result is due to Svenonius [42] and is a generalization of the first. We state these results for the first order predicate language with identity $L(\mu)$ but trivial modifications yield similar results for first order predicate languages without identity.

If $\Omega$ is a set of formulae (of $L(\mu)$ ), we denote by $\bar{\Omega}$ the smallest set of formulae containing $\Omega$ together with all formulae " $i=j$ " for $i$ and $j \in I$ and closed under all propositional connectives and quantifiers.

For any formula $H$ with free variables $\left\{i_{1}, \cdots, i_{n}\right\}$, and system $\left\langle X, R_{\lambda}\right\rangle$ we call the interpretation (or abstract) of $H$ in the system $X$, the set of all $\left(x_{1}, \cdots, x_{n}\right) \in X^{n}$ such that for some (and hence all) $x \in X^{I}$ such that $x_{i_{1}}=x_{1}, \cdots, x_{i_{n}}=x_{n}, H$ is satisfied for the assignment $x$.

The statement of Beth's theorem which follows is adapted from Theorem 2 in [3].

THEOREM 11.1. Let $\Sigma$ be a consistent set of formulae (of the language $L(\mu)$ ) and let $\Omega$ be a set of formulae and $G_{0}$ a formula with free variables $\left\{i_{1}, \cdots, i_{n}\right\}$. Suppose that for any two models $\left\langle X, R_{\lambda}\right\rangle$ and $\left\langle X, S_{\lambda}\right\rangle$ of $\Sigma$ in which the interpretations of any formula in $\Omega$ coincide, the interpretations of $G_{0}$ also coincide. Then there exists a formula $H \in \bar{\Omega}$ (containing free at most the variables $\left.\left\{i_{1}, \cdots, i_{n}\right\}\right)$ and such that $\left(\forall_{i_{1}}\right) \cdots\left(\forall_{i_{n}}\right)\left(G_{0} \equiv H\right)$ is a consequence of $\Sigma$.

Proof. Let $A_{1}=F_{\mu} / \tilde{\Sigma}$ and $A$ be the equality subalgebra generated by $\{G / \tilde{\Sigma} \mid G \in \Omega\}$. If we let $p=G_{0} / \tilde{\Sigma}$, the desired conclusion is that $p \in A$. Assume $\rho \notin A$. Then by the equality version of Theorem 4.2 and the representation theorem for equality algebras, there exist two reduced representations $\left[f_{i}, A, X, 0\right], i=1,2$ such that $f_{1}\left|A=f_{2}\right| A$ and $f_{1}(p) \neq f_{2}(p)$. By Theorem 10.1 these yield two models with the same domain in which the abstracts of all $G \in \Omega$ agree but those of $G_{0}$ do not.

Q.E.D.

Svenonius' theorem reads as follows.

THeOREM 11.2. Let $\Sigma, \Omega$ and $G_{0}$ be as in the preceding theorem. Then the following are equivalent:

(i) There does not exist finitely many formulae $G_{1}, \cdots, G_{s}$ of $\bar{\Omega}$ such that

$$
\bigvee_{i=1}^{s}\left(\forall_{i_{1}}\right) \cdots\left(\forall_{i_{n}}\right)\left(G_{0} \equiv G_{i}\right) \in \tilde{\Sigma} .
$$

(ii) For some model $\left\langle X, R_{\lambda}\right\rangle$ of $\Sigma$ and some permutation $\phi$ of $X$, the interpretations of all $G \in \Omega$ in $\left\langle X, R_{\lambda}\right\rangle$ and in the isomorphic model $\left\langle X, \phi R_{\lambda}\right\rangle$ coincide while those of $G_{0}$ differ. 
Proof. That (ii) implies (i) is obvious. We prove that (i) implies (ii). Let $A_{1}, A$ and $p$ be as in the proof of Beth's theorem. It is a simple consequence of the semisimplicity of polyadic algebras that, if $A_{1}$ is an extension of an algebra $A$ and $p \in A_{1}$, then no relation of the form

$$
\bigvee_{i=1}^{s}(\forall I)\left(p \equiv p_{i}\right)=1
$$

for some $p_{1}, \cdots, p_{s}$ in $A$ holds iff for some surjective homomorphism $h: A_{1} \rightarrow \bar{A}_{1}$ with $\bar{A}_{1}$ simple, we have $\bar{p}=h(p) \notin h(A)$. Set $\bar{A}=h(A)$ and let, by the equality version of 4.3 (and 2.3) $D$ be a rich simple equality extension of $\bar{A}_{1}$ and $\rho$ be an $\bar{A}$-automorphism of $D$ such that $\rho(\bar{p}) \neq \bar{p}$. Let $[f, D, X, 0]$ be the canonical representation and let $\left[f_{i}, A_{1}, X, 0\right], i=1,2$, be defined by $f_{1}=f h$ and $f_{2}=f \rho h$. Denoting by $\phi$ the permutation of $X$ induced by $\rho$, we have that $\phi$ is an isomorphism of the model $\left\langle X,\left(f_{1} F_{\lambda} / T\right)^{*}\right\rangle$ onto the model $\left\langle X,\left(f_{2} F_{\lambda} / T\right)^{*}\right\rangle$ and the nterpretations of all formulae in $\Omega$ coincide in these two models of $\Sigma$ whereas those of $G_{0}$ differ.

Q.E.D.

We see that Theorem 4.2 and Proposition 4.3 are algebraic counterparts of Beth's theorem and of Svenonius' theorem respectively.

12. Elementary equivalence. We shall next combine the results of Chapter I on homogeneous extensions of polyadic algebras with those of Chapter II on generalized ultrapowers to obtain a characterization of the concept of elementary equivalence closely related to results of S.B. Kochen and H. J. Keisler.

THEOREM 12.1. For any set $H$ of (not necessarily similar) relational systems such that $|H| \leqq \gamma$ and $|X| \leqq \alpha$ for all systems $X \in H(\gamma, \alpha$ infinite cardinals $)$ there exists a cardinal $\beta$ such that $\alpha \leqq \beta \leqq \gamma 2^{\alpha}$ and an ultrafilter $D$ of $\mathscr{P}_{\omega}\left(\alpha^{\beta}\right)\left({ }^{12}\right)$ such that:

(i) For any system with domain $X$ in $H$ the generalized ultrapower $F\left(\alpha^{\beta}, X\right) / D$ has cardinality at most $\beta$ and exactly $\beta$ if $|X|=\alpha$;

(ii) Any two similar systems $\left\langle X_{1}, R_{\lambda}\right\rangle$ and $\left\langle X_{2}, S_{\lambda}\right\rangle$ in $H$ are elementarily equivalent iff the generalized ultrapower systems $\left\langle F\left(\alpha^{\beta}, X_{1}\right) / D, R^{+} \lambda\right\rangle$ and $\left\langle F\left(\alpha^{\beta}, X_{2}\right) / D, S_{\lambda}^{+}\right\rangle$are isomorphic.

Proof. e immediately note that the "if" part of (ii) is obvious since the systems $X_{1}$ and $X_{2}$ can be elementarily imbedded into the generalized ultrapower systems.

Let $\bar{M}$ be a rich simple homogeneous extension of the full algebra $C_{\alpha}$ (Theorem 2.11). For each system $X \in H$ choose a stretch imbedding $s: C_{X} \rightarrow C_{\alpha}$ such that if $|X|=\alpha, s$ is an epimorphism and hence is equality preserving. Let $A_{1}$ and $A_{2}$

(12) Here " $\alpha$ " does not stand for an ordinal but for the set of all functions from the set $\beta$ into the set $\alpha$. 
be the algebras of two elementarily equivalent systems $\left\langle X_{1}, R_{\lambda}\right\rangle$ and $\left\langle X_{2}, S_{\lambda}\right\rangle$ in $H$ respectively, and let $\sigma: A_{1} \rightarrow A_{2}$ be the isomorphism that implements that equivalence. For each such pair of systems choose an automorphism $\rho$ of $\bar{M}$ extending the monomorphism $s_{2} \sigma s_{1}^{-1}$ of $s_{1} A_{1}$ onto $s_{2} A_{2}, s_{1}$ and $s_{2}$ being the stretch imbeddings belonging to $X_{1}$ and $X_{2}$ respectively. Let $G$ be the group of automorphisms of $\bar{M}$ generated by these chosen automorphisms. Of course $|G| \leqq \gamma$; and, by Lemma 5.1 , we have $\left|C_{\alpha}\right|=2^{\alpha}$ assuming, as we may, that $|I|=\omega$. By Theorem 5.4 , let $M$ be a rich extension of $C_{\alpha}$ contained in $\bar{M}$, invariant under $G$, and such that $|M| \leqq \gamma 2^{\alpha}$. Denote by $Z$ the set of all constants of $M$ and by $Y$ the set of all constants of $C_{\alpha}(Z)$. As $C_{\alpha}(Z)$ has an equality we have that $\alpha \leqq|Y| \leqq \gamma 2^{\alpha}$. Set $\beta=|Y|$.

By Theorem 8.4, if for $X \in H, g$ is the canonical representation of $\left(s C_{X}\right)(Y)$, the representation $g s$ is equivalent to a representation $\left[f_{D}, C_{X}, F\left(\alpha^{\beta}, X\right) / D, 0\right]$ in which $D$ is determined only by $C_{\alpha}(Y)$. As the constants of $\left(s C_{X}\right)(Y)$ correspond to equivalence classes of elements of $Y$ we have that $\left|F\left(\alpha^{\beta}, X\right) / D\right| \leqq|Y|$. If $|X|=\alpha$, these classes are precisely the singletons $\{y\}$ for $y \in Y$ by virtue of the choice of $s$, so that, in this case, the equality holds. This completes the proof of (i).

As to (ii), since $\rho$ maps $\left(s_{1} A_{1}\right)(Y)$ onto $\left(s_{2} A_{2}\right)(Y)$ in such a way that $\rho\left(s_{1} \hat{R}_{\lambda}\right)=s_{2} \hat{S}_{\lambda}$ (for all $\lambda$ ), $\rho$ induces a biunique map $\bar{\rho}$ from $F\left(\alpha^{\beta}, X_{1}\right) / D$ onto $F\left(\alpha^{\beta}, X_{2}\right) / D$ such that $\bar{\rho} R_{\lambda}^{+}=S_{\lambda}^{+}$. This means that $\bar{\rho}$ is an isomorphism between the generalized ultrapower structures mentioned in (ii).

Q.E.D.

In this proof, essential use is made of the concept of polyadic algebra without equality; if for all $X \in H,|X|=\alpha$ then instead of invoking Theorem 2.11 we may invoke the equality version of this theorem so that $\bar{M}$ is an equality extension of $C_{\alpha}$.

For the $H$ of Theorem 12.1 one can take, for instance, a set of representatives from all isomorphism classes of relational systems of cardinality $\leqq$ some infinite cardinal $\alpha$ and of types involving at most $\alpha$ relations. Then according to Lemma 5.2 we can set $\gamma=2^{\alpha}$ and the generalized ultrapowers of the theorem are of cardinality at most $2^{\alpha}$.

By letting $H$ in Theorem 12.1 consist of two similar relational systems with an arbitrary number of relations, we obtain

COROLlaRY 12.2. Let $\left\langle X_{1}, R_{\lambda}\right\rangle$ and $\left\langle X_{2}, S_{\lambda}\right\rangle$ be two similar infinite relational systems and let $\alpha$ be the larger of $\left|X_{1}\right|$ and $\left|X_{2}\right|$. Then the two systems are elementarily equivalent iff for some cardinal $\beta$ such that $\alpha \leqq \beta \leqq 2^{\alpha}$, and some ultrafilter $D$ in $\mathscr{P}_{\omega}\left(\alpha^{\beta}\right)$ the generalized ultrapower systems $\left\langle F\left(\alpha^{\beta}, X_{1}\right) / D, R_{\lambda}^{+}\right\rangle$and $\left\langle F\left(\alpha^{\beta}, X_{2}\right) / D, S_{\lambda}^{+}\right\rangle$are isomorphic and of cardinality $\beta$.

A comparison between the above results and similar ones of S. B. Kochen and of H. J. Keisler is in order. These authors have both defined concepts of limit ultrapowers which are known to be equivalent (see Kochen $[30 ; 32]$ and Keisler 
$[23 ; 24 ; 26 ; 27])\left({ }^{13}\right)$. Our Theorem 12.1 should be compared with Theorem 9.6 of [32]; it will be seen that the cardinality conditions of our result presents an advantage over those stated on p. 243 of [32](14). The above Corollary 12.2 must be compared with the first theorem of [24] supplemented by Theorem 4 of [27].

Both Kochen (see Theorem 1 in [31])(15) and Keisler (see [25], Theorem 5 in [28], and Corollary A.8 in [29]) have announced the possibility of characterizing the concept of elementary equivalence by means of ultrapowers only. Despite its obvious merits, this result, aside from requiring the use of the continuum hypothesis, has the further slight disadvantage that the isomorphic ultrapowers of two given elementarily equivalent systems have a cardinality which depends on the number of relations of their type in addition to the cardinality of the given systems.

13. Elementary homogeneity. We conclude this paper by proving a result of R. L. Vaught (see Theorem 2 in [45] and Theorem A.1 in [29]). The proof uses the continuum hypothesis as it is based on a result of $\S 6$. To state Vaught's theorem we introduce some definitions.

For any cardinal number $\beta$, a relational system $X$ is said to be $\beta$-elementarily universal if any elementarily equivalent system $Y$ such that $|Y| \leqq \beta$, is isomorphic to an elementary subsystem of $X$. The system $X$ is said to be $\beta$-elementarily homogeneous if any isomorphism $\sigma: X_{1} \rightarrow X_{2}$ between two elementary subsystems of $X$ of cardinality less than $\beta$ can be extended to an automorphism of $X$. Vaught's theorem can be stated as follows.

THEOREM 13.1. For any complete theory $T$ in $L(\mu)$ and infinite cardinal $\alpha$ such that $\alpha \geqq|\Lambda|$ where $\Lambda$ is the domain of the type $\mu$, there exists, up to isomorphism, exactly one $\alpha^{+}$-elementarily universal and homogeneous model $X$ of $T$ of cardinality $\alpha^{+}$.

Proof. Let $A$ be the simple algebra $F(\mu) / T$ and assume that $|I|=\omega$. Then $\alpha \geqq|A|$ and the theorem follows directly from Theorem 10.1 , Corollary 10.2 , Theorem 10.4 and Theorem 6.6.

Q.E.D.

(13) The equivalence of our generalized ultrapowers with Keisler's limit ultrapowers can be obtained by comparing our Theorem 8.1 with Theorem 2 of [26]. Also a generalized ultrapower $F\left(W^{K}, Y\right) / D$ can be shown to be isomorphic with the direct limit of a canonical direct system of ultrapowers as defined in [26] by letting the directed set $X$ there be the set of all finite subsets of $K$ ordered by inclusion.

(14) The class $K$ mentioned in the upper part of p. 247 of [32] must be further restricted by putting a bound on the number of relations involved in the types of systems in $K$, in order to make it a set. This assumption was probably made tacitly there.

(15) As to this reference, see also footnotes 5 and 8 in [29] and footnote 15 in [32]. 


\section{BIBLIOGRAPHY}

1. E. Beth, On Padoa's method in the theory of definition, Indag. Math. 56 (1953), 330-339.

2. A. Church, Introduction to mathematical logic, Vol. 1, Princeton Univ. Press, Princeton, N. J., 1956.

3. W. Craig, Three uses of the Herbrand-Gentzen theorem in relating model theory to proof theory, J. Symbolic Logic 22 (1957), 269-285.

4. A. Daigneault, Products of polyadic algebras and their representations, Doctoral dissertation, Princeton Univ., 1959.

5. ——, Extension of isomorphisms of polyadic algebras, Notices Amer. Math. Soc. 8 (1961), 609.

6. _- Generalized ultrapowers, Notices Amer. Math. Soc. 8 (1961), 628.

7. A. Daigneault and D. Monk, Representation theory for polyadic algebras, Fund. Math. 52 (1963), 151-176.

8. T. Frayne, D. Scott and A. Tarski, Reduced products, Notices Amer. Math. Soc. 5 (1958), 673.

9. T. Frayne, A. Morel and D. Scott, Reduced direct products, Fund. Math. 51 (1962), 195-228.

10. B. A. Galler, Cylindric and polyadic algebras, Proc. Amer. Math. Soc. 8 (1957), 176-183.

11. P. R. Halmos, The basic concepts of algebraic logic, Amer. Math. Monthly 53 (1956), 363-387.

12. - Algebraic logic. I. Monadic Boolean algebras, Comp. Math. 12 (1955), 217-249.

13. — Algebraic logic. II. Homogeneous locally finite polyadic Boolean algebras of infinite degree, Fund. Math. 43 (1956), 255-325.

14. ——, Algebraic logic. III. Predicates, terms, and operations in polyadic algebras, Trans. Amer. Math. Soc. 83 (1956), 430-470.

15. - Algebraic logic. IV. Equality in polyadic algebras, Trans. Amer. Math. Soc. 86 (1957), 1-27.

16. —, Algebraic logic, Chelsea, New York, 1962.

17. - Polyadic algebras, Summaries of talks presented at the Summer Institute of Symbolic Logic, Ithaca, N.Y. 1957, pp. 252-256.

18. __ Boolean algebras, Mimeographed, Chicago, Ill., 1959.

19. L. Henkin, La structure algébrique des théories mathématiques, Gauthier-Villars, Paris, 1956.

20. L. Henkin and A. Tarski, Cylindric algebras, Proc. Sympos. Pure Math. Vol. II, pp. 83-113, Amer. Math. Soc., Providence, R.I., 1961.

21. B. Jónsson, Universal relational systems, Math. Scand. 4 (1956), 193-208.

22. - Homogeneous universal relational systems, Math. Scand. 8 (1960), 137-142.

23. H. J. Keisler, Limit reduced products, Notices Amer. Math. Soc. 6 (1959), 520.

24. - A mathematical characterization of elementary equivalence, Notices Amer. Math. Soc. 6 (1959), 520

25. —_, Isomorphism of ultraproducts, Notices Amer. Math. Soc. 7 (1960), 70-71.

26. - On the class of limit ultrapowers of a relational system, Notices Amer. Math. Soc. 7 (1960), 878 .

27. - Cardinalities of ultrapowers and a theorem of Rabin, Notices Amer. Math. Soc. 7 (1960), 879.

28. — Isomorphism of ultraproducts. II, Notices Amer. Math. Soc. 8 (1961), 63-64.

29. __ Ultraproducts and elementary classes, Nederl. Akad. Wetensch. Proc. Ser. A 64 (1961), 477-495. 
30. S. B. Kochen, An algebraic characterization of arithmetical equivalence, Notices Amer. Math. Soc. 6 (1959), 437.

31. - The isomorphism of ultrapowers of elementarily equivalent systems, Notices Amer. Math. Soc. 7 (1960), 971.

32. - Ultraproducts in the theory of models, Ann. of Math. (2) 74 (1961), 221-261.

33. M. Krasner, Une généralisation de la notion de corps, J. Math. Pures Appl. (9) 17 (1938), 367-385.

34. - Généralisation abstraite de la théorie de Galois, Colloques internationaux du Centre National de la Recherche Scientifique no. 24, 1950, pp. 163-168.

35. —_ Les algebres cylindriques, Bull. Soc. Math. France 86 (1959), 315-319.

36. M. L'Abbé, Structures algébriques suggérées par la logique mathématique, Bull. Socs Math. France 86 (1959), 299-314.

37. J.Los, Quelques remarques, théorèmes et problèmes sur les classes définissable. d'algebres, Mathematical interpretations of formal systems, pp. 98-113, North-Holland, Amsterdam, 1955.

38. A. Robinson, A result on consistency and its application to the theory of definition, Nederl. Akad. Wetensch. Proc. Ser. A 59 (1956), 47-58.

39. R. Sikorski, Products of abstract algebras, Fund. Math. 39 (1952), 211-228.

40. Th. Skolem, Peano's axioms and models of arithmetic, Mathematical interpretations of formal systems, pp. 1-14, North-Holland, Amsterdam, 1955.

41. M. H. Stone, Some algebraic aspects of logic, Mimeographed, Univ. of Chicago, Chicago, Ill., 1956.

42. L. Svenonius, $A$ theorem on permutations in models, Theoria 25 (1959), 173-178.

43. A. Tarski, Some notions and methods on the border-line of algebra and metamathematics, Proc. Internat. Congr. Math. (Cambridge, Mass., 1950) Vol. 1, pp. 705-720, Amer. Math. Soc., Providence, R. I., 1952.

44. A. Tarski and R. L. Vaught, Arithmetical extensions of relational systems, Comp. Math. 13 (1957), 81-102.

45. R. L. Vaught, Homogeneous universal models of complete theories, Notices Amer. Math. Soc. 5 (1958), 775.

\section{UNIVERSITÉ DE MONTRÉAL, Montréal, Canada}

